\title{
Full Rank Solutions for the MIMO Gaussian Wiretap Channel With an Average Power Constraint
}

\author{
S. Ali. A. Fakoorian, Student Member, IEEE, and A. Lee Swindlehurst, Fellow, IEEE
}

\begin{abstract}
This paper considers a multiple-input multipleoutput (MIMO) Gaussian wiretap channel with a transmitter, a legitimate receiver and an eavesdropper, each equipped with multiple antennas. We first study the rank of the optimal input covariance matrix that achieves the secrecy capacity of the MIMO Gaussian wiretap channel under an average power constraint. The rank and other properties of the optimal solution are derived based on certain relationships between the channel matrices for the legitimate receiver and eavesdropper. Next, by obtaining necessary and sufficient conditions on the MIMO wiretap channel parameters, we determine the conditions under which the optimal input covariance matrix is full-rank or rank-deficient. For the case that the optimal input covariance is full-rank, we fully characterize the solution. When the optimal input covariance is rank-deficient, we show that the given MIMO wiretap channel can be modeled by an equivalent wiretap channel whose optimal input covariance is full rank and achieves the same secrecy capacity as the original system. Numerical results are presented to illustrate the proposed theoretical findings.
\end{abstract}

Index Terms-MIMO Wiretap Channel, physical layer security, secrecy capacity.

\section{INTRODUCTION}

T HE broadcast nature of a wireless medium makes it very susceptible to eavesdropping, where the transmitted message is decoded by unintended receiver(s). Recent informationtheoretic research on secure communication has focused on enhancing security at the physical layer. The wiretap channel, first introduced and studied by Wyner [1], is the most basic physical layer model that captures the problem of communication security. Wyner showed that when an eavesdropper's channel is a degraded version of the main channel, the source and destination can achieve a positive secrecy rate, while ensuring that the eavesdropper gets zero bits of information. The maximum secrecy rate from the source to the destination is defined as the secrecy capacity. The Gaussian wiretap channel, in which the outputs at the legitimate receiver and at the eavesdropper are corrupted by additive white Gaussian noise, was studied in [2].

Manuscript received August 05, 2012; revised December 14, 2012 and February 26, 2013; accepted February 26, 2013. Date of publication March 21, 2013; date of current version April 25, 2013. The associate editor coordinating the review of this manuscript and approving it for publication was Prof. Ana Perez-Neira. This work was supported by the U.S. Army Research Office under the Multi-University Research Initiative (MURI) grant W911NF-07-1-0318, and by the National Science Foundation under grant CCF-1117983.

The authors are with the Center for Pervasive Communications and Computing, University of California, Irvine, CA 92697-2625 USA (e-mail: afakoori@uci.edu; swindle@uci.edu).

Color versions of one or more of the figures in this paper are available online at http://ieeexplore.ieee.org.

Digital Object Identifier 10.1109/TSP.2013.2253774
Determining the secrecy capacity of a general multiple-input multiple-output (MIMO) Gaussian wiretap channel is in general a difficult non-convex optimization problem, and has been addressed independently in [3]-[5]. Oggier and Hassibi [3] and Khisti and Wornell [4] followed an indirect approach using a Sato-like argument and matrix analysis tools. They considered the problem of finding the secrecy capacity of the Gaussian MIMO wiretap channel under the average total power constraint, and a closed-form expression for the secrecy capacity in the high signal-to-noise-ratio (SNR) regime was obtained in [4]. In [5], Liu and Shamai propose a more information-theoretic approach using the enhancement concept, originally presented by Weingarten et al. [6], as a tool for the characterization of the MIMO Gaussian broadcast channel capacity. Liu and Shamai have shown that an enhanced degraded version of the channel attains the same secrecy capacity as does a Gaussian input distribution. From the mathematical solution in [5] it was evident that such an enhanced channel exists; however it was not clear how to construct such a channel until the work of [7], which provided a closed-form expression for the secrecy capacity under an input covariance matrix constraint. While this result is interesting since the expression for the secrecy capacity is valid for all SNR scenarios, in principle an exhaustive search would be required to obtain the secrecy capacity for the MIMO Gaussian wiretap channel under an average total power constraint.

In [8], Zhang et al. attempted to bypass the non-convex optimization of the optimal input covariance matrix by drawing connections to a sequence of convex cognitive radio transmission problems, and obtained upper and lower bounds on the MIMO secrecy capacity under the average power constraint. Optimal power allocation methods for the GSVD-based precoding scheme were presented in [9], although in general these are not capacity-achieving. In [10], an iterative approach based on a fixed point algorithm is proposed for the MIMO wiretap channel under the average power constraint, but the convergence of the algorithm is not guaranteed. Except for some special cases of the MIMO wiretap channel, where the solution has rank one or all nodes have two antennas [10]-[12], the optimal input covariance matrix that achieves the secrecy capacity under the average total power constraint is still unknown.

Given the increased design flexibility that the average power constraint provides compared with the covariance matrix power constraint, which places considerable limits on the per-antenna power and transmit correlation structure, it is clearly desirable to study this case more fully. To this end, we focus in this paper on cases where the optimal transmit covariance matrix for the general MIMO Gaussian wiretap channel is full rank under the 
average power constraint. In particular, we obtain necessary and sufficient conditions under which the optimal transmit covariance matrix is full rank. When the solution is full-rank, we characterize the optimal covariance in closed form. When the solution is not full rank, we show that there exists an equivalent MIMO wiretap channel that achieves the same secrecy capacity with an input covariance that is full rank. An earlier version of the results in this paper was presented in [13]. After the development of this work, we noted that another paper independently derived the optimal full-rank transmit covariance using a different approach [14], assuming that the eavesdropper's channel is full-column rank. As we show later in the paper, this assumption is not required for an optimal full-rank transmit covariance matrix. Also, we provide additional insight on the structure of the transmit covariance matrix when the optimal solution is rank deficient.

The rest of this paper is organized as follows. In the next section, we describe the assumed mathematical model and revisit the solution for the wiretap channel under the input covariance matrix constraint. The rank property of the optimal input covariance matrix under the average power constraint is investigated in Section III, and in Section IV we characterize the conditions under which the input covariance matrix that achieves the secrecy capacity of a wiretap channel under the average power constraint is full-rank. Some interesting facts regarding the optimal solution are also discussed in Section IV. In Section V we present numerical results to illustrate our results. Finally, Section VI concludes the paper.

Notation: Throughout the paper, we use boldface uppercase letters to denote matrices. Vector-valued random variables are written with non-boldface uppercase letters (e.g., $X$ ), while the corresponding boldface lowercase letter $(\mathbf{x})$ denotes a specific realization of the random variable. Scalar variables are written with non-boldface (lowercase or uppercase) letters. The Hermitian (i.e., conjugate) transpose is denoted by $(.)^{H}$, the matrix trace by $\operatorname{Tr}($.$) , and \mathbf{I}$ indicates an identity matrix. Inequality $\mathbf{A} \preceq$ $\mathbf{B}$ means that $\mathbf{A}-\mathbf{B}$ is Hermitian positive semi-definite. Mutual information between the random variables $A$ and $B$ is denoted by $I(A ; B), E$ is the expectation operator, and $\mathcal{C N}\left(0, \sigma^{2}\right)$ represents the complex circularly symmetric Gaussian distribution with zero mean and variance $\sigma^{2}$. The orthogonal projection onto the column space of $\mathbf{X}$ is denoted by $\mathbf{P}_{\mathbf{X}}=\mathbf{X}\left(\mathbf{X}^{H} \mathbf{X}\right)^{-1} \mathbf{X}^{H}$, and $\mathbf{P}_{\mathbf{X}}^{\perp}=\mathbf{I}-\mathbf{P}_{\mathbf{X}}$ denotes the projection onto the space orthogonal to $\mathbf{X} \cdot \operatorname{span}\{\mathbf{X}\}$ denotes the space spanned by the column vectors of $\mathbf{X}$, and $\operatorname{span}\{\mathbf{X}\}^{\perp}$ denotes the space orthogonal to $\operatorname{span}\{\mathbf{X}\}$.

\section{System MOdel AND PRIOR WORK}

We begin with a multiple-antenna wiretap channel with $n_{t}$ transmit antennas and $n_{r}$ and $n_{e}$ receive antennas at the legitimate recipient and the eavesdropper, respectively:

$$
\begin{aligned}
& \mathbf{y}_{r}=\mathbf{H} \mathbf{x}+\mathbf{z}_{r} \\
& \mathbf{y}_{e}=\mathbf{G x}+\mathbf{z}_{e}
\end{aligned}
$$

where $\mathbf{x}$ is a zero-mean $n_{t} \times 1$ transmitted signal vector, $\mathbf{z}_{r} \in$ $\mathbb{C}^{n_{r} \times 1}$ and $\mathbf{z}_{e} \in \mathbb{C}^{n_{e} \times 1}$ are additive white Gaussian noise vec- tors at the receiver and eavesdropper, respectively, with i.i.d. entries distributed as $\mathcal{C N}(0,1)$. The matrices $\mathbf{H} \in \mathbb{C}^{n_{r} \times n_{t}}$ and $\mathbf{G} \in \mathbb{C}^{n_{e} \times n_{t}}$ represent the channels associated with the receiver and the eavesdropper, respectively. Similar to other papers considering the perfect secrecy rate of the wiretap channel, we assume that the transmitter has perfect channel state information (CSI) for both the legitimate receiver and the eavesdropper. For the Gaussian channel, where Gaussian inputs are an optimal choice, the secrecy capacity $\mathcal{C}_{s e c}$ is the maximum value of the secrecy rate [3]

$$
\begin{aligned}
R(\mathbf{Q}) & =I\left(X ; Y_{r}\right)-I\left(X ; Y_{e}\right) \\
& =\log \left|\mathbf{H} \mathbf{Q} \mathbf{H}^{H}+\mathbf{I}\right|-\log \left|\mathbf{G Q} \mathbf{G}^{H}+\mathbf{I}\right|
\end{aligned}
$$

where $\mathbf{Q}=E\left\{\mathbf{x x}^{H}\right\}$ is the input covariance matrix. Constraints on $\mathbf{Q}$ are obviously required in order to obtain a meaningful secrecy capacity. As described below, the secrecy capacity problem has been considered under the following two constraints: a strict covariance matrix constraint of the form $\mathbf{Q} \preceq \mathbf{S}$, or a less restrictive constraint on the average transmitted power $\operatorname{Tr}(\mathbf{Q}) \leq P_{t}$.

In [7], the secret communication problem was analyzed under the input covariance constraint, defined as

$$
\begin{aligned}
\mathcal{C}_{\text {sec }}(\mathbf{S})= & \max \log \left|\mathbf{H} \mathbf{Q} \mathbf{H}^{H}+\mathbf{I}\right|-\log \left|\mathbf{G} \mathbf{Q} \mathbf{G}^{H}+\mathbf{I}\right| \\
& \text { s.t. } \quad \mathbf{Q} \preceq \mathbf{S}
\end{aligned}
$$

where $\mathbf{S}$ is a positive semi-definite matrix that defines the constraint. An explicit expression for the secrecy capacity under (4) was obtained by applying the generalized eigenvalue decomposition to the following two positive definite matrices

$$
\left(\mathbf{S}^{\frac{1}{2}} \mathbf{H}^{H} \mathbf{H} \mathbf{S}^{\frac{1}{2}}+\mathbf{I}, \quad \mathbf{S}^{\frac{1}{2}} \mathbf{G}^{H} \mathbf{G} \mathbf{S}^{\frac{1}{2}}+\mathbf{I}\right) .
$$

In particular, there exists an invertible generalized eigenvector matrix $\mathbf{C}$ such that [16]

$$
\begin{aligned}
& \mathbf{C}^{H}\left[\mathbf{S}^{\frac{1}{2}} \mathbf{G}^{H} \mathbf{G} \mathbf{S}^{\frac{1}{2}}+\mathbf{I}\right] \mathbf{C}=\mathbf{I} \\
& \mathbf{C}^{H}\left[\mathbf{S}^{\frac{1}{2}} \mathbf{H}^{H} \mathbf{H} \mathbf{S}^{\frac{1}{2}}+\mathbf{I}\right] \mathbf{C}=\mathbf{\Lambda}
\end{aligned}
$$

where $\boldsymbol{\Lambda}=\operatorname{diag}\left\{\lambda_{1}, \ldots, \lambda_{n_{t}}\right\}$ is a positive definite diagonal matrix and $\lambda_{1}, \ldots, \lambda_{n_{t}}$ represent the generalized eigenvalues. Without loss of generality, we assume the eigenvalues are ordered as

$$
\lambda_{1} \geq \ldots \geq \lambda_{b}>1 \geq \lambda_{b+1} \geq \ldots \geq \lambda_{n_{t}}>0
$$

so that a total of $b\left(0 \leq b \leq n_{t}\right)$ are greater than 1 . Hence, we can write $\boldsymbol{\Lambda}$ as

$$
\boldsymbol{\Lambda}=\left[\begin{array}{cc}
\boldsymbol{\Lambda}_{1} & \mathbf{0} \\
\mathbf{0} & \boldsymbol{\Lambda}_{2}
\end{array}\right]
$$

where $\boldsymbol{\Lambda}_{1}=\operatorname{diag}\left\{\lambda_{1}, \ldots, \lambda_{b}\right\}$ and $\boldsymbol{\Lambda}_{2}=$ $\operatorname{diag}\left\{\lambda_{b+1}, \ldots, \lambda_{n_{t}}\right\}$. We can partition $\mathbf{C}$ similarly:

$$
\mathbf{C}=\left[\begin{array}{ll}
\mathbf{C}_{1} & \mathbf{C}_{2}
\end{array}\right]
$$

where $\mathbf{C}_{1}$ is the $n_{t} \times b$ submatrix representing the generalized eigenvectors corresponding to $\left\{\lambda_{1}, \ldots, \lambda_{b}\right\}$ and $\mathbf{C}_{2}$ is the $n_{t} \times$ 
$\left(n_{t}-b\right)$ submatrix representing the generalized eigenvectors corresponding to $\left\{\lambda_{b+1}, \ldots, \lambda_{n_{t}}\right\}$.

Using the above notation, the secrecy capacity of the MIMO wiretap channel under the input covariance constraint (4) can be expressed as [7], [15, Theorem 3]:

$$
\mathcal{C}_{\text {sec }}(\mathbf{S})=\sum_{i=1}^{b} \log \lambda_{i}=\log \left|\boldsymbol{\Lambda}_{\mathbf{1}}\right|
$$

where the optimal input covariance matrix $\mathbf{Q}_{S}^{*}$ that maximizes (4) and attains (10) is given by

$$
\mathbf{Q}_{S}^{*}=\mathbf{S}^{\frac{1}{2}} \mathbf{C}\left[\begin{array}{cc}
\left(\mathbf{C}_{1}^{H} \mathbf{C}_{1}\right)^{-1} & \mathbf{0} \\
\mathbf{0} & \mathbf{0}
\end{array}\right] \mathbf{C}^{H} \mathbf{S}^{\frac{1}{2}}
$$

Remark 1: From (6) and (7), one can easily confirm that if $\mathbf{H}^{H} \mathbf{H} \preceq \mathbf{G}^{H} \mathbf{G}$, then for any $\mathbf{S} \succeq \mathbf{0}$ we have $\boldsymbol{\Lambda} \preceq \mathbf{I}$. In other words, in this case the pencil in (5) has no generalized eigenvalue bigger than 1 . Thus, $\mathcal{C}_{\text {sec }}(\mathbf{S})=0$ for any $\mathbf{S} \succeq \mathbf{0}$.

Lemma 1: When $\mathbf{H}^{H} \mathbf{H} \succ \mathbf{G}^{H} \mathbf{G}$, for any $n_{t} \times n_{t}$ matrix $\mathbf{S} \succeq \mathbf{0}$, all the generalized eigenvalues of the pencil $\left(\mathbf{S}^{\frac{1}{2}} \mathbf{H}^{H} \mathbf{H S}^{\frac{1}{2}}+\mathbf{I}, \mathbf{S}^{\frac{1}{2}} \mathbf{G}^{H} \mathbf{G S}^{\frac{1}{2}}+\mathbf{I}\right)$ are strictly bigger than 1 $(\boldsymbol{\Lambda} \succ \mathbf{I})$ iff $\mathbf{S}$ is full rank, i.e., $\mathbf{S} \succ \mathbf{0}$.

Proof: Subtracting (6) from (7), a straightforward computation yields

$$
\mathbf{S}^{\frac{1}{2}}\left(\mathbf{H}^{H} \mathbf{H}-\mathbf{G}^{H} \mathbf{G}\right) \mathbf{S}^{\frac{1}{2}}=\mathbf{C}^{-H}(\boldsymbol{\Lambda}-\mathbf{I}) \mathbf{C}^{-1} .
$$

When $\mathbf{H}^{H} \mathbf{H} \succ \mathbf{G}^{H} \mathbf{G}$, both sides of the above equality are positive semi-definite; i.e., for any $\mathbf{S} \succeq \mathbf{0}$ we must have $\boldsymbol{\Lambda} \succeq \mathbf{I}$. If $\mathbf{S} \succ \mathbf{0}$, the left side is full-rank and thus $\boldsymbol{\Lambda} \succ \mathbf{I}$, and vice versa.

In this paper, we consider the secrecy capacity problem in (2) under the more common average power constraint:

$$
\begin{gathered}
\mathcal{C}_{\text {sec }}\left(P_{t}\right)=\max _{\mathbf{Q} \succeq \mathbf{0}} \log \left|\mathbf{H Q} \mathbf{H}^{H}+\mathbf{I}\right|-\log \left|\mathbf{G Q} \mathbf{G}^{H}+\mathbf{I}\right| \\
\text { s.t. } \operatorname{Tr}\left(E\left\{\mathbf{x} \mathbf{x}^{H}\right\}\right)=\operatorname{Tr}(\mathbf{Q}) \leq P_{t} .
\end{gathered}
$$

The average power constraint is a much less restrictive constraint that provides considerable additional flexibility in increasing the secrecy rate of the MIMO wiretap channel. For this constraint, no computable secrecy capacity expression has been derived to date for the general MIMO case. It was shown in [6, Lemma 1], [15] that the wiretap channel under the average power constraint (12) can, at least in principle, be found through an exhaustive search of the solution to (4) over the set $\{\mathbf{S}: \mathbf{S} \succeq \mathbf{0}, \operatorname{Tr}(\mathbf{S}) \leq P\}$ :

$$
\mathcal{C}_{\text {sec }}\left(P_{t}\right)=\max _{\mathbf{S} \succeq \mathbf{0}, \operatorname{Tr}(\mathbf{S}) \leq P_{t}} \mathcal{C}_{\text {sec }}(\mathbf{S}),
$$

where for any given semidefinite $\mathbf{S}, \mathcal{C}_{\text {sec }}(\mathbf{S})$ should be computed as in (10). Also note that for any covariance constraint $\mathbf{S}$, the optimal input covariance matrix $\mathbf{Q}_{S}^{*}$ that attains $\mathcal{C}_{\text {sec }}(\mathbf{S})$ is given by (11), and $\mathbf{Q}_{S}^{*} \preceq \mathbf{S}$.

In the next section, we investigate the rank of the optimal input covariance matrix $\mathbf{Q}^{*}$ that attains $\mathcal{C}_{\text {sec }}\left(P_{t}\right)$. In Section IV, we obtain the optimal $\mathbf{Q}^{*}$ under the average power constraint for the case that $\mathbf{Q}^{*}$ is full-rank.

\section{Properties of the Optimal Solution UNDER AN Average Power Constraint}

In the following, we exclude the special case $\mathbf{H}^{H} \mathbf{H} \preceq \mathbf{G}^{H} \mathbf{G}$ for which $C_{s e c}$ is trivially 0 for any $\mathbf{S} \succeq \mathbf{0}$, and consequently for any $P_{t}$, as pointed out in Remark 1 . Also we note that problem (12) under $\operatorname{Tr}(\mathbf{Q}) \leq P_{t}$ is equivalent to the problem under $\operatorname{Tr}(\mathbf{Q})=P_{t}$, as discussed in [3, Eq. (9)], [10]. Considering (12) in the form of (13), and recalling that $\mathbf{Q}_{S}^{*} \preceq \mathbf{S}$ for any $\mathbf{S} \succeq 0$, then $\operatorname{Tr}\left(\mathbf{Q}_{S}^{*}\right) \leq \operatorname{Tr}(\mathbf{S})$ and the above point implies that we can restrict the set of input covariance constraints in (13) to $\mathbf{S} \succeq \mathbf{0}$, $\operatorname{Tr}(\mathbf{S})=P_{t}$.

Let $\widehat{\mathbf{S}}$ denote the input covariance constraint that maximizes (13), and let $\mathbf{Q}^{*} \equiv \mathbf{Q}_{\widehat{S}}^{*}$ represent the corresponding optimal input covariance matrix that according to (11) attains $\mathcal{C}_{\text {sec }}\left(P_{t}\right)$ :

$$
\mathbf{Q}^{*}=\widehat{\mathbf{S}}^{\frac{1}{2}} \widehat{\mathbf{C}}\left[\begin{array}{cc}
\left(\widehat{\mathbf{C}}_{1}^{H} \widehat{\mathbf{C}}_{1}\right)^{-1} & \mathbf{0} \\
\mathbf{0} & \mathbf{0}
\end{array}\right] \widehat{\mathbf{C}}^{H} \widehat{\mathbf{S}}^{\frac{1}{2}},
$$

where $\widehat{\mathbf{C}}$ and $\widehat{\mathbf{C}}_{1}$ have respectively the same definitions as those of $\mathbf{C}$ and $\mathbf{C}_{1}$, given by (6)-(9), but here for the pencil $\left(\widehat{\mathbf{S}}^{\frac{1}{2}} \mathbf{H}^{H} \mathbf{H} \widehat{\mathbf{S}}^{\frac{1}{2}}+\mathbf{I}, \widehat{\mathbf{S}}^{\frac{1}{2}} \mathbf{G}^{H} \mathbf{G} \widehat{\mathbf{S}}^{\frac{1}{2}}+\mathbf{I}\right)$. Note that $\mathbf{Q}^{*}$ can be rewritten as

$$
\begin{aligned}
\mathbf{Q}^{*} & =\widehat{\mathbf{S}}^{\frac{1}{2}}\left[\begin{array}{ll}
\widehat{\mathbf{C}}_{1} & \widehat{\mathbf{C}}_{2}
\end{array}\right]\left[\begin{array}{cc}
\left(\widehat{\mathbf{C}}_{1}^{H} \widehat{\mathbf{C}}_{1}\right)^{-1} & \mathbf{0} \\
\mathbf{0} & \mathbf{0}
\end{array}\right]\left[\begin{array}{c}
\widehat{\mathbf{C}}_{1}^{H} \\
\widehat{\mathbf{C}}_{2}^{H}
\end{array}\right] \widehat{\mathbf{S}}^{\frac{1}{2}} \\
& =\widehat{\mathbf{S}}^{\frac{1}{2}} \widehat{\mathbf{C}}_{1}\left(\widehat{\mathbf{C}}_{1}^{H} \widehat{\mathbf{C}}_{1}\right)^{-1} \widehat{\mathbf{C}}_{1}^{H} \widehat{\mathbf{S}}^{\frac{1}{2}} \\
& =\widehat{\mathbf{S}}^{\frac{1}{2}} \mathbf{P}_{\widehat{\mathbf{C}}_{1}} \widehat{\mathbf{S}}^{\frac{1}{2}}
\end{aligned}
$$

where $\mathbf{P}_{\widehat{\mathbf{C}}_{1}}=\widehat{\mathbf{C}}_{1}\left(\widehat{\mathbf{C}}_{1}^{H} \widehat{\mathbf{C}}_{1}\right)^{-1} \widehat{\mathbf{C}}_{1}^{H}$ is the orthogonal projection onto the columns of $\widehat{\mathbf{C}}_{1}$. Moreover, let $\mathbf{P} \widehat{\widehat{\mathbf{C}}}_{1}=\mathbf{I}-\mathbf{P}_{\widehat{\mathbf{C}}_{1}}$ be the projection onto the space orthogonal to $\widehat{\mathbf{C}}_{1}$. We have

$$
\begin{aligned}
\operatorname{Tr}\left(\mathbf{Q}^{*}\right) & =\operatorname{Tr}\left(\widehat{\mathbf{S}}^{\frac{1}{2}} \mathbf{P}_{\widehat{\mathbf{C}}_{1}} \widehat{\mathbf{S}}^{\frac{1}{2}}\right) \\
& =\operatorname{Tr}\left(\widehat{\mathbf{S}} \mathbf{P}_{\widehat{\mathbf{C}}_{1}}\right) \\
& =\operatorname{Tr}\left(\widehat{\mathbf{S}} \mathbf{P}_{\widehat{\mathbf{C}}_{1}} \mathbf{P}_{\widehat{\mathbf{C}}_{1}}\right) \\
& =\operatorname{Tr}\left(\mathbf{P}_{\widehat{\mathbf{C}}_{1}} \widehat{\mathbf{S P}}_{\widehat{\mathbf{C}}_{1}}\right)
\end{aligned}
$$

where (16) comes from the fact that for any $\mathbf{A} \in \mathbb{C}^{a \times b}$ and $\mathbf{B} \in$ $\mathbb{C}^{b \times a}, \operatorname{Tr}(\mathbf{A B})=\operatorname{Tr}(\mathbf{B A})$, and (17) holds because $\mathbf{P}_{\widehat{\mathbf{C}}_{1}}=$ $\mathbf{P}_{\widehat{\mathbf{C}}_{1}} \mathbf{P}_{\widehat{\mathbf{C}}_{1}}$. Similarly we have

$$
\begin{aligned}
\operatorname{Tr}(\widehat{\mathbf{S}}) & =\operatorname{Tr}\left(\left(\mathbf{P}_{\widehat{\mathbf{C}}_{1}}+\mathbf{P}_{\widehat{\mathbf{C}}_{1}}^{\perp}\right) \widehat{\mathbf{S}}\left(\mathbf{P}_{\widehat{\mathbf{C}}_{1}}+\mathbf{P}_{\widehat{\mathbf{C}}_{1}}^{\perp}\right)\right) \\
& =\operatorname{Tr}\left(\mathbf{P}_{\widehat{\mathbf{C}}_{1}} \widehat{\mathbf{S}}_{\widehat{\mathbf{C}}_{1}}\right)+\operatorname{Tr}\left(\mathbf{P}_{\widehat{\mathbf{C}}_{1}}^{\perp} \widehat{\mathbf{S}} \mathbf{P}_{\widehat{\mathbf{C}}_{1}}^{\perp}\right) \\
& =\operatorname{Tr}\left(\mathbf{Q}^{*}\right)+\operatorname{Tr}\left(\mathbf{P}_{\widehat{\mathbf{C}}_{1}}^{\perp} \widehat{\mathbf{S}} \mathbf{P}_{\widehat{\mathbf{C}}_{1}}^{\perp}\right)
\end{aligned}
$$

where in (19) we used the fact that $\operatorname{Tr}(\mathbf{A B})=\operatorname{Tr}(\mathbf{B A})$ and $\mathbf{P}_{\widehat{\mathrm{C}}_{1}} \mathbf{P}_{\widehat{\mathrm{C}}_{1}}^{\perp}=\mathbf{0}$, and where (20) results from (18).

Lemma 2: For the optimal $\widehat{\mathbf{S}}$, we have $\operatorname{span}\left\{\widehat{\mathbf{C}}_{1}\right\}=$ $\operatorname{span}\{\widehat{\mathbf{S}}\}$.

Proof: The proof is obtained using (20), and by noting that for the optimal $\widehat{\mathbf{S}}$ we must have $\operatorname{Tr}(\widehat{\mathbf{S}})=\operatorname{Tr}\left(\mathbf{Q}^{*}\right)=P_{t}$. 
This means that we must have $\operatorname{Tr}\left(\mathbf{P}{\widehat{\widehat{\mathbf{C}}_{1}}}_{\mathbf{S P}^{-}} \widehat{\mathrm{C}}_{1}\right)=0$, or equivalently $\mathbf{P} \widehat{\mathbf{C}}_{1} \widehat{\mathbf{S}}=\mathbf{0}$, which consequently shows that $\operatorname{span}\{\widehat{\mathbf{S}}\} \subseteq$ $\operatorname{span}\left\{\widehat{\mathbf{C}}_{1}\right\}$. To complete the proof we only need to show that $\operatorname{span}\left\{\widehat{\mathbf{C}}_{1}\right\} \subseteq \operatorname{span}\{\widehat{\mathbf{S}}\}$.

Let $\widehat{\mathbf{c}}_{1}$ denote a column vector of $\widehat{\mathbf{C}}_{1}$, i.e., $\widehat{\mathbf{c}}_{1}$ represents a generalized eigenvector of the pencil $\left(\widehat{\mathbf{S}}^{\frac{1}{2}} \mathbf{H}^{H} \mathbf{H} \widehat{\mathbf{S}}^{\frac{1}{2}}+\mathbf{I}, \widehat{\mathbf{S}}^{\frac{1}{2}} \mathbf{G}^{H} \mathbf{G} \widehat{\mathbf{S}}^{\frac{1}{2}}+\mathbf{I}\right)$ corresponding to a generalized eigenvalue bigger than 1 :

$$
\frac{\widehat{\mathbf{c}}_{1}^{H}\left(\widehat{\mathbf{S}}^{\frac{1}{2}} \mathbf{H}^{H} \mathbf{H} \widehat{\mathbf{S}}^{\frac{1}{2}}+\mathbf{I}\right) \widehat{\mathbf{c}}_{1}}{\widehat{\mathbf{c}}_{1}^{H}\left(\widehat{\mathbf{S}}^{\frac{1}{2}} \mathbf{G}^{H} \mathbf{G} \widehat{\mathbf{S}}^{\frac{1}{2}}+\mathbf{I}\right) \widehat{\mathbf{c}}_{1}}>1 .
$$

We can write $\widehat{\mathbf{c}}_{1}=\mathbf{P}_{\widehat{\mathbf{S}}} \widehat{\mathbf{c}}_{1}+\mathbf{P}_{\widehat{\mathrm{S}}}^{\perp} \widehat{\mathbf{c}}_{1}$, where $\mathbf{P}_{\widehat{\mathrm{S}}}=\widehat{\mathbf{S}}\left(\widehat{\mathbf{S}}^{H} \widehat{\mathbf{S}}^{-1} \widehat{\mathbf{S}}^{H}\right.$ is the projection onto the column span of $\widehat{\mathbf{S}}$, and $\mathbf{P} \widehat{\widehat{S}}_{\mathrm{S}}^{\perp}=\mathbf{I}-$ $\mathbf{P}_{\widehat{\mathrm{S}}}$ is the projection onto the orthogonal space. Letting $a=$ $\widehat{\mathbf{c}}_{1}^{H} \widehat{\mathbf{S}}^{\frac{1}{2}} \mathbf{H}^{H} \mathbf{H} \widehat{\mathbf{S}}^{\frac{1}{2}} \widehat{\mathbf{c}}_{1}$ and $d=\widehat{\mathbf{c}}_{1}^{H} \widehat{\mathbf{S}}^{\frac{1}{2}} \mathbf{G}^{H} \mathbf{G} \widehat{\mathbf{S}}^{\frac{1}{2}} \widehat{\mathbf{c}}_{1}$, we note that $a>d$, and the only component of $\widehat{\mathbf{c}}_{1}$ that affects the values of $a$ and $d$ is $\mathbf{P}_{\widehat{\mathrm{S}}} \widehat{\mathbf{c}}_{1}$. Now, one can easily show that when $a>d \geq 0$, for any $x \geq 0, y \geq 0$, we have

$$
\frac{a+x}{d+x} \geq \frac{a+x+y}{d+x+y}
$$

where equality holds if $y=0$. Noting that $\widehat{\mathbf{c}}_{1}^{H} \widehat{\mathbf{c}}_{1}=\widehat{\mathbf{c}}_{1}^{H} \mathbf{P}_{\widehat{\mathbf{s}}} \widehat{\mathbf{c}}_{1}+$ $\widehat{\mathbf{c}}_{1}^{H} \mathbf{P} \widehat{\widehat{\mathbf{S}}}^{\perp} \widehat{\mathbf{c}}_{1}=x+y$, the above point implies that the generalized eigenvalue corresponding to $\widehat{\mathbf{c}}_{1}$ is maximized when $\mathbf{P} \stackrel{\perp}{\triangle} \widehat{\mathbf{c}}_{1}=\mathbf{0}$. Consequently, for the optimal $\widehat{\mathbf{S}}$ we must have $\mathbf{P} \widehat{\stackrel{\mathrm{S}}{\perp}} \widehat{\mathbf{C}}_{1}=\mathbf{0}$, i.e., $\operatorname{span}\left\{\widehat{\mathbf{C}}_{1}\right\} \subseteq \operatorname{span}\{\widehat{\mathbf{S}}\}$, which completes the proof. One can also show $\operatorname{span}\left\{\widehat{\mathbf{C}}_{1}\right\} \subseteq \operatorname{span}\{\widehat{\mathbf{S}}\}$ by recalling that $\widehat{\mathbf{C}}_{1}$ corresponds to eigenvalues bigger than 1 and the goal of $\widehat{\mathbf{S}}$ is to maximize the product of such eigenvalues (see (10)); thus a component in $\widehat{\mathbf{C}}_{1}$ which is orthogonal to span $\{\widehat{\mathbf{S}}\}$ would be useless.

Let $\widehat{b}=\operatorname{rank}(\widehat{\mathbf{S}})$. We note from Lemma 2 that $\operatorname{rank}(\widehat{\mathbf{S}})=$ $\operatorname{rank}\left(\widehat{\mathbf{C}}_{1}\right)=\operatorname{rank}\left(\widehat{\boldsymbol{\Lambda}}_{1}\right)=\widehat{b}$, so $\widehat{b}$ represents the number of generalized eigenvalues of $\left(\widehat{\mathbf{S}}^{\frac{1}{2}} \mathbf{H}^{H} \mathbf{H} \widehat{\mathbf{S}}^{\frac{1}{2}}+\mathbf{I}, \widehat{\mathbf{S}}^{\frac{1}{2}} \mathbf{G}^{H} \mathbf{G} \widehat{\mathbf{S}}^{\frac{1}{2}}+\mathbf{I}\right)$ that are strictly greater than 1 . Denote the singular value decomposition of $\widehat{\mathbf{C}}_{1}$ as

$$
\widehat{\mathbf{C}}_{1}=\mathbf{U}_{c} \mathbf{D}_{c} \mathbf{V}_{c}^{H},
$$

where $\mathbf{U}_{c}$ is a $n_{t} \times \widehat{b}$ matrix whose columns are an orthonormal basis for $\operatorname{span}\left\{\widehat{\mathbf{C}}_{1}\right\}, \mathbf{U}_{c}^{H} \mathbf{U}_{c}=\mathbf{I}, \mathbf{D}_{c}$ is a $\widehat{b} \times \widehat{b}$ positive definite diagonal matrix, and $\mathbf{V}_{c}$ is a unitary matrix. From (21), one can easily confirm that $\mathbf{P}_{\widehat{\mathbf{C}}_{1}}=\widehat{\mathbf{C}}_{1}\left(\widehat{\mathbf{C}}_{1}^{H} \widehat{\mathbf{C}}_{1}\right)^{-1} \widehat{\mathbf{C}}_{1}=\mathbf{U}_{c} \mathbf{U}_{c}^{H}$. Recall from Lemma 2 that $\operatorname{span}\left\{\widehat{\mathbf{C}}_{1}\right\}=\operatorname{span}\{\widehat{\mathbf{S}}\}$, so that $\widehat{\mathbf{S}}$ can be written as

$$
\widehat{\mathbf{S}}=\mathbf{U}_{c} \mathbf{Q}_{e q} \mathbf{U}_{c}^{H},
$$

where $\mathbf{Q}_{e q} \in \mathbb{C}^{\widehat{b} \times \widehat{b}}$ is a positive definite matrix. Using (22) in (15), and noting that $\widehat{\mathbf{S}}^{\frac{1}{2}}=\mathbf{U}_{c} \mathbf{Q}_{e q}^{\frac{1}{2}} \mathbf{U}_{c}^{H}$, we have

$$
\begin{aligned}
\mathbf{Q}^{*} & =\mathbf{U}_{c} \mathbf{Q}_{e q}^{\frac{1}{2}} \mathbf{U}_{c}^{H} \mathbf{U}_{c} \mathbf{U}_{c}^{H} \mathbf{U}_{c} \mathbf{Q}_{e q}^{\frac{1}{2}} \mathbf{U}_{c}^{H} \\
& =\mathbf{U}_{c} \mathbf{Q}_{e q} \mathbf{U}_{c}^{H}=\widehat{\mathbf{S}}
\end{aligned}
$$

The following lemma reveals another property of the optimal input covariance matrix under the average power constraint.

Lemma 3: For the optimal $\widehat{\mathbf{S}}=\mathbf{Q}^{*}$, the pencil $\left(\widehat{\mathbf{S}}^{\frac{1}{2}} \mathbf{H}^{H} \mathbf{H} \widehat{\mathbf{S}}^{\frac{1}{2}}+\mathbf{I}, \widehat{\mathbf{S}}^{\frac{1}{2}} \mathbf{G}^{H} \mathbf{G} \widehat{\mathbf{S}}^{\frac{1}{2}}+\mathbf{I}\right)$ has no generalized eigenvalue less than one.

Proof: Recall from the discussion prior to (21) that $\widehat{b}$ denotes the number of generalized eigenvalues of the pencil $\left(\widehat{\mathbf{S}}^{\frac{1}{2}} \mathbf{H}^{H} \mathbf{H} \widehat{\mathbf{S}}^{\frac{1}{2}}+\mathbf{I}, \widehat{\mathbf{S}}^{\frac{1}{2}} \mathbf{G}^{H} \mathbf{G} \widehat{\mathbf{S}}^{\frac{1}{2}}+\mathbf{I}\right)$ that are strictly bigger than 1 . We show that the remaining $n_{t}-\widehat{b}$ generalized eigenvalues, if any, are equal to one. From Lemma 2 , we have $\operatorname{rank}(\widehat{\mathbf{S}})=\operatorname{rank}\left(\widehat{\mathbf{C}}_{1}\right)=\widehat{b}$, where $\widehat{\mathbf{C}}_{1}$ is a $n_{t} \times \widehat{b}$ matrix representing generalized eigenvectors corresponding to generalized eigenvalues bigger than 1 . Now define a $n_{t} \times n_{t}-\widehat{b}$ matrix $\widehat{\mathbf{C}}_{2}=\left[\widehat{\mathbf{c}}_{21} \ldots \widehat{\mathbf{c}}_{2 n_{t}-\widehat{b}}\right]$ that represents an orthonormal basis for $\operatorname{span}\{\widehat{\mathbf{S}}\}^{\perp}$. Note that these basis vectors will satisfy $\widehat{\mathbf{S}}^{\frac{1}{2}} \widehat{\mathbf{c}}_{2 i}=0$, and thus they will also be equal to generalized eigenvectors of $\left(\widehat{\mathbf{S}}^{\frac{1}{2}} \mathbf{H}^{H} \mathbf{H} \widehat{\mathbf{S}}^{\frac{1}{2}}+\mathbf{I}, \widehat{\mathbf{S}}^{\frac{1}{2}} \mathbf{G}^{H} \mathbf{G} \widehat{\mathbf{S}}^{\frac{1}{2}}+\mathbf{I}\right)$ with generalized eigenvalues equal to 1 :

$$
\left(\widehat{\mathbf{S}}^{\frac{1}{2}} \mathbf{H}^{H} \mathbf{H} \widehat{\mathbf{S}}^{\frac{1}{2}}+\mathbf{I}\right) \widehat{\mathbf{c}}_{2 i}=1 \times\left(\widehat{\mathbf{S}}^{\frac{1}{2}} \mathbf{G}^{H} \mathbf{G} \widehat{\mathbf{S}}^{\frac{1}{2}}+\mathbf{I}\right) \widehat{\mathbf{c}}_{2 i} .
$$

Thus, for the optimal $\widehat{\mathbf{S}}$, the pencil $\left(\widehat{\mathbf{S}}^{\frac{1}{2}} \mathbf{H}^{H} \mathbf{H} \widehat{\mathbf{S}}^{\frac{1}{2}}+\mathbf{I}, \widehat{\mathbf{S}}^{\frac{1}{2}} \mathbf{G}^{H} \mathbf{G} \widehat{\mathbf{S}}^{\frac{1}{2}}+\mathbf{I}\right)$ will have $\widehat{b}$ generalized eigenvalues greater than 1 and $n_{t}-\widehat{b}$ generalized eigenvalues equal to 1 :

$$
\begin{aligned}
& \widehat{\mathbf{C}}^{H}\left[\widehat{\mathbf{S}}^{\frac{1}{2}} \mathbf{H}^{H} \mathbf{H} \widehat{\mathbf{S}}^{\frac{1}{2}}+\mathbf{I}\right] \widehat{\mathbf{C}}=\left[\begin{array}{cc}
\widehat{\boldsymbol{\Lambda}}_{1} & \mathbf{0} \\
\mathbf{0} & \mathbf{I}
\end{array}\right] \\
& \widehat{\mathbf{C}}^{H}\left[\widehat{\mathbf{S}}^{\frac{1}{2}} \mathbf{G}^{H} \mathbf{G} \widehat{\mathbf{S}}^{\frac{1}{2}}+\mathbf{I}\right] \widehat{\mathbf{C}}=\left[\begin{array}{ll}
\mathbf{I} & \mathbf{0} \\
\mathbf{0} & \mathbf{I}
\end{array}\right]
\end{aligned}
$$

where $\widehat{\mathbf{C}}=\left[\widehat{\mathbf{C}}_{1} \widehat{\mathbf{C}}_{2}\right]$ is the generalized eigenvector matrix, and $\widehat{\Lambda}_{1}$ is a $\widehat{b} \times \widehat{b}$ diagonal matrix with diagonal elements representing generalized eigenvalues greater that 1 .

In the following, another property of the optimal $\mathbf{Q}^{*}$ is revealed. Using (24), a straightforward computation yields

$$
\widehat{\mathbf{C}}^{H} \widehat{\mathbf{S}}^{\frac{1}{2}}\left[\mathbf{H}^{H} \mathbf{H}-\mathbf{G}^{H} \mathbf{G}\right] \widehat{\mathbf{S}}^{\frac{1}{2}} \widehat{\mathbf{C}}=\left[\begin{array}{cc}
\widehat{\boldsymbol{\Lambda}}_{1}-\mathbf{I} & \mathbf{0} \\
\mathbf{0} & \mathbf{0}
\end{array}\right] \succeq \mathbf{0},
$$

from which we have

$$
\widehat{\mathbf{C}}_{1}^{H} \widehat{\mathbf{S}}^{\frac{1}{2}}\left[\mathbf{H}^{H} \mathbf{H}-\mathbf{G}^{H} \mathbf{G}\right] \widehat{\mathbf{S}}^{\frac{1}{2}} \widehat{\mathbf{C}}_{1}=\widehat{\boldsymbol{\Lambda}}_{1}-\mathbf{I} \succ \mathbf{0} .
$$

Using (21) and (22) in (25), we have

$$
\mathbf{V}_{c} \mathbf{D}_{c} \mathbf{Q}_{e q}^{\frac{1}{2}} \mathbf{U}_{c}^{H}\left[\mathbf{H}^{H} \mathbf{H}-\mathbf{G}^{H} \mathbf{G}\right] \mathbf{U}_{c} \mathbf{Q}_{e q}^{\frac{1}{2}} \mathbf{D}_{c} \mathbf{V}_{c}^{H}=\widehat{\boldsymbol{\Lambda}}_{\mathbf{1}}-\mathbf{I} \succ \mathbf{0},
$$

which results in

$$
\begin{aligned}
\mathbf{U}_{c}^{H}\left[\mathbf{H}^{H} \mathbf{H}\right. & \left.-\mathbf{G}^{H} \mathbf{G}\right] \mathbf{U}_{c} \\
& =\mathbf{Q}_{e q}^{-\frac{1}{2}} \mathbf{D}_{c}^{-1} \mathbf{V}_{c}^{H}\left(\widehat{\boldsymbol{\Lambda}}_{1}-\mathbf{I}\right) \mathbf{V}_{c} \mathbf{D}_{c}^{-1} \mathbf{Q}_{e q}^{-\frac{1}{2}} \succ \mathbf{0} .
\end{aligned}
$$

The above equality shows that for the optimal $\mathbf{Q}^{*}$ with $\widehat{b}=$ $\operatorname{rank}\left(\mathbf{Q}^{*}\right), \mathbf{U}_{c}^{H}\left[\mathbf{H}^{H} \mathbf{H}-\mathbf{G}^{H} \mathbf{G}\right] \mathbf{U}_{c}$ is a $\widehat{b} \times \widehat{b}$ positive definite matrix. 
Theorem 1: For the optimal $\mathbf{Q}^{*}=\widehat{\mathbf{S}}$, we have

$$
\operatorname{rank}\left(\mathbf{Q}^{*}\right) \leq m
$$

where $m$ is the number of positive eigenvalues of the matrix $\mathbf{H}^{H} \mathbf{H}-\mathbf{G}^{H} \mathbf{G}$.

Proof: The proof is obtained using (26) and by contradiction. Please refer to Appendix A for details.

Remark 2: From Theorem 1, one can easily confirm that the optimal $\mathbf{Q}^{*}$ can be full rank only in the case that $m=n_{t}$, i.e. $\mathbf{H}^{H} \mathbf{H} \succ \mathbf{G}^{H} \mathbf{G}$. For all other scenarios, the optimal $\mathbf{Q}^{*}$ will be low rank. From a MIMO channel modeling perspective, the condition $\mathbf{H}^{H} \mathbf{H} \succ \mathbf{G}^{H} \mathbf{G}$ means that the channel to the legitimate receiver dominates that of the eavesdropper across all subchannels; in other words, the eavesdropper's channel is degraded with respect to the legitimate receiver for any precoder chosen by the transmitter.

\section{Characterization OF THE Optimal InPUT Covariance}

In this section, we characterize the secrecy capacity under the average power constraint for a particular class of MIMO Gaussian wiretap channels where the optimal solution $\mathbf{Q}^{*}$ is full rank. While necessary conditions for a full-rank $\mathbf{Q}^{*}$ were characterized in the previous section, here we derive sufficient conditions as well. We also provide some details on how to handle the case where $\mathbf{Q}^{*}$ is rank deficient.

We begin by rewriting problem (12) here:

$\mathcal{C}_{s e c}\left(P_{t}\right)=\max _{\mathbf{Q} \succeq \mathbf{0}, \operatorname{Tr}(\mathbf{Q})=P_{t}} \log \left|\mathbf{H} \mathbf{Q} \mathbf{H}^{H}+\mathbf{I}\right|-\log \left|\mathbf{G Q} \mathbf{Q G}^{H}+\mathbf{I}\right|$.

The Lagrangian associated with this problem is given by

$\mathcal{L}=\log \left|\mathbf{H Q H} \mathbf{H}^{H}+\mathbf{I}\right|-\log \left|\mathbf{G Q G} \mathbf{Q}^{H}+\mathbf{I}\right|-\mu\left(\operatorname{Tr}(\mathbf{Q})-P_{t}\right)$

$$
+\operatorname{Tr}(\mathbf{M Q})
$$

where $\mu>0$ and $\mathbf{M} \succeq \mathbf{0}$ are the Lagrange multipliers. The optimal $\mathbf{Q}^{*}$ must satisfy the following KKT conditions:

$$
\begin{aligned}
& \mathbf{H}^{H}\left(\mathbf{H Q}^{*} \mathbf{H}^{H}+\mathbf{I}\right)^{-1} \mathbf{H}-\mathbf{G}^{H}\left(\mathbf{G Q}^{*} \mathbf{G}^{H}+\mathbf{I}\right)^{-1} \mathbf{G} \\
& \quad=\mu \mathbf{I}-\mathbf{M} \\
& \mu\left(\operatorname{Tr}\left(\mathbf{Q}^{*}\right)-P_{t}\right)=0 \\
& \mathbf{Q}^{*} \mathbf{M}=\mathbf{M Q}^{*}=\mathbf{0} .
\end{aligned}
$$

Using the matrix inversion lemma [16], (30) can be written as

$$
\left(\mathbf{H}^{H} \mathbf{H} \mathbf{Q}^{*}+\mathbf{I}\right)^{-1} \mathbf{H}^{H} \mathbf{H}-\mathbf{G}^{H} \mathbf{G}\left(\mathbf{Q}^{*} \mathbf{G}^{H} \mathbf{G}+\mathbf{I}\right)^{-1}=\mu \mathbf{I}-\mathbf{M} .
$$

Left multiplication by $\left(\mathbf{H}^{H} \mathbf{H} \mathbf{Q}^{*}+\mathbf{I}\right)$ and right multiplication by $\left(\mathbf{Q}^{*} \mathbf{G}^{H} \mathbf{G}+\mathbf{I}\right)$ of both sides of (33) yields

$$
\begin{aligned}
\mathbf{H}^{H} \mathbf{H}-\mathbf{G}^{H} \mathbf{G} & =\mu\left(\mathbf{H}^{H} \mathbf{H} \mathbf{Q}^{*}+\mathbf{I}\right)\left(\mathbf{Q}^{*} \mathbf{G}^{H} \mathbf{G}+\mathbf{I}\right)-\mathbf{M} \\
& =\mu\left(\mathbf{G}^{H} \mathbf{G Q}^{*}+\mathbf{I}\right)\left(\mathbf{Q}^{*} \mathbf{H}^{H} \mathbf{H}+\mathbf{I}\right)-\mathbf{M},
\end{aligned}
$$

where in obtaining (34) we have used the KKT condition (32), and (35) comes from the fact that (34) is Hermitian.

\section{A. Full-Rank Solution}

Here we focus on solving (28) for the case that $\mathbf{H}^{H} \mathbf{H} \succ$ $\mathbf{G}^{H} \mathbf{G}$, since this is the necessary condition for having a full- rank optimal $\mathbf{Q}^{*}$. As we characterize the full rank $\mathbf{Q}^{*}$, the sufficient conditions are revealed as well.

Remark 3: By following exactly the same steps as in the proof of [3, Proposition 5], one can easily show that for the case of $\mathbf{H}^{H} \mathbf{H} \succ \mathbf{G}^{H} \mathbf{G}$, the optimization problem (28) is convex ${ }^{1}$ in Q.

Thus for the case of interest, the KKT conditions (31), (32) and (34) are necessary and sufficient conditions for the optimality of $\mathbf{Q}^{*}$. In other words, any $\mathbf{Q} \succeq \mathbf{0}$ that satisfies those conditions is an optimal solution for the problem (28). By the KKT condition (32), a full rank $\mathbf{Q}^{*}$ implies that $\mathbf{M}=\mathbf{0}$. Thus, (34) and (35) simplify to

$$
\begin{aligned}
\mathbf{H}^{H} \mathbf{H}-\mathbf{G}^{H} \mathbf{G} & =\mu\left(\mathbf{H}^{H} \mathbf{H} \mathbf{Q}^{*}+\mathbf{I}\right)\left(\mathbf{Q}^{*} \mathbf{G}^{H} \mathbf{G}+\mathbf{I}\right) \\
& =\mu\left(\mathbf{G}^{H} \mathbf{G Q}^{*}+\mathbf{I}\right)\left(\mathbf{Q}^{*} \mathbf{H}^{H} \mathbf{H}+\mathbf{I}\right) .
\end{aligned}
$$

Lemma 4: Let the diagonal matrix $\mathbf{D}$ and the unitary matrix $\boldsymbol{\Phi}_{\overline{\mathrm{s}}}$ respectively denote the eigenvalue and eigenvector matrices of $\left(\overline{\mathbf{S}}^{\frac{1}{2}} \mathbf{G}^{H} \mathbf{G} \overline{\mathbf{S}}^{\frac{1}{2}}+\mathbf{I}\right)$, where we set $\overline{\mathbf{S}}=\left(\mathbf{H}^{H} \mathbf{H}-\mathbf{G}^{H} \mathbf{G}\right)^{-1}$ :

$$
\left(\overline{\mathbf{S}}^{\frac{1}{2}} \mathbf{G}^{H} \mathbf{G} \overline{\mathbf{S}}^{\frac{1}{2}}+\mathbf{I}\right)=\boldsymbol{\Phi}_{\overline{\mathbf{s}}} \mathbf{D} \boldsymbol{\Phi}_{\overline{\mathbf{s}}}^{H} .
$$

Then we have

$$
\begin{aligned}
\mathbf{H}^{H} \mathbf{H} & =\overline{\mathbf{S}}^{-\frac{1}{2}} \boldsymbol{\Phi}_{\overline{\mathbf{S}}} \mathbf{D} \boldsymbol{\Phi}_{\overline{\mathbf{s}}}^{H} \overline{\mathbf{S}}^{-\frac{1}{2}} \\
\mathbf{G}^{H} \mathbf{G} & =\overline{\mathbf{S}}^{-\frac{1}{2}} \boldsymbol{\Phi}_{\overline{\mathbf{S}}}(\mathbf{D}-\mathbf{I}) \boldsymbol{\Phi}_{\overline{\mathbf{S}}}^{H} \overline{\mathbf{S}}^{-\frac{1}{2}} .
\end{aligned}
$$

Proof: Eq. (40) comes directly from (38). Please refer to Appendix B for details on obtaining (39).

It is clear from (40) that $\mathbf{D} \succeq \mathbf{I}$. Also we note that, if $\mathbf{G}^{H} \mathbf{G} \succ$ $\mathbf{0}$, then $\mathbf{D}-\mathbf{I} \succ \mathbf{0}$ and vice versa. As we will observe in Theorem 2, it is not necessary for $\mathbf{G}^{H} \mathbf{G}$ to be full rank in order to have a full-rank optimal input covariance matrix $\mathbf{Q}^{*}$. While we assume throughout the paper and without loss of generality that the diagonal matrix $\mathbf{D}-\mathbf{I}$ is invertible, for the case of rank deficient $\mathbf{G}^{H} \mathbf{G}$ one can follow the calculations below by substituting $\epsilon>0$ for the zero-diagonal elements of $\mathbf{D}-\mathbf{I}$ and letting $\epsilon \downarrow 0$ (see Lemma 7), where the $\downarrow$ symbol means $\epsilon$ decreases to zero from above.

Using (39) and (40) in (36), after some simplification we have

$$
\begin{aligned}
\overline{\mathbf{S}}^{-\frac{1}{2}} \boldsymbol{\Phi}_{\overline{\mathbf{s}}} \boldsymbol{\Phi}_{\overline{\mathbf{s}}}^{H} \overline{\mathbf{S}}^{-\frac{1}{2}}= & \mu\left(\overline{\mathbf{S}}^{-\frac{1}{2}} \boldsymbol{\Phi}_{\overline{\mathbf{S}}} \mathbf{D} \boldsymbol{\Phi}_{\overline{\mathbf{S}}}^{H} \overline{\mathbf{S}}^{-\frac{1}{2}} \mathbf{Q}^{*}+\mathbf{I}\right) \\
& \times\left(\mathbf{Q}^{*} \overline{\mathbf{S}}^{-\frac{1}{2}} \boldsymbol{\Phi}_{\overline{\mathbf{s}}}(\mathbf{D}-\mathbf{I}) \boldsymbol{\Phi}_{\overline{\mathbf{s}}}^{H} \overline{\mathbf{S}}^{-\frac{1}{2}}+\mathbf{I}\right) \\
= & \mu \overline{\mathbf{S}}^{-\frac{1}{2}} \boldsymbol{\Phi}_{\overline{\mathbf{s}}}\left(\mathbf{D} \boldsymbol{\Phi}_{\overline{\mathbf{s}}}^{H} \overline{\mathbf{S}}^{-\frac{1}{2}} \mathbf{Q}^{*}+\boldsymbol{\Phi}_{\overline{\mathbf{s}}}^{H} \overline{\mathbf{S}}^{\frac{1}{2}}\right) \\
& \times\left(\mathbf{Q}^{*} \overline{\mathbf{S}}^{-\frac{1}{2}} \boldsymbol{\Phi}_{\overline{\mathbf{S}}}(\mathbf{D}-\mathbf{I})+\overline{\mathbf{S}}^{\frac{1}{2}} \boldsymbol{\Phi}_{\overline{\mathbf{s}}}\right) \boldsymbol{\Phi}_{\overline{\mathbf{s}}}^{H} \overline{\mathbf{S}}^{-\frac{1}{2}} \\
= & \mu \overline{\mathbf{S}}^{-\frac{1}{2}} \boldsymbol{\Phi}_{\overline{\mathbf{S}}}(\mathbf{D} \mathbf{W}+\mathbf{I}) \boldsymbol{\Phi}_{\overline{\mathbf{s}}}^{H} \overline{\mathbf{S}} \boldsymbol{\Phi}_{\overline{\mathbf{s}}} \\
& \times(\mathbf{W}(\mathbf{D}-\mathbf{I})+\mathbf{I}) \boldsymbol{\Phi}_{\overline{\mathbf{s}}}^{H} \overline{\mathbf{S}}^{-\frac{1}{2}}
\end{aligned}
$$

where in (41) we defined

$$
\mathbf{W}=\boldsymbol{\Phi}_{\overline{\mathbf{S}}}^{H} \overline{\mathbf{S}}^{-\frac{1}{2}} \mathbf{Q}^{*} \overline{\mathbf{S}}^{-\frac{1}{2}} \boldsymbol{\Phi}_{\overline{\mathbf{S}}} .
$$

From (41), we obtain

$$
\mathbf{I}=\mu(\mathbf{D W}+\mathbf{I}) \boldsymbol{\Phi}_{\overline{\mathbf{s}}}^{H} \overline{\mathbf{S}} \boldsymbol{\Phi}_{\overline{\mathbf{s}}}(\mathbf{W}(\mathbf{D}-\mathbf{I})+\mathbf{I}) .
$$

${ }^{1}$ In fact, the optimization problem (28) is convex in $\mathbf{Q}$ when $\mathbf{H}^{H} \mathbf{H} \succeq$ $\mathbf{G}^{H} \mathbf{G}$. 
Define the diagonal matrix $\mathbf{X}=\left(\mathbf{I}-\mathbf{D}^{-1}\right)^{\frac{1}{2}}$. Note that the definition of $\mathbf{X}$ implies that $(\mathbf{X D ~ W})^{H}=\mathbf{W D X}=\mathbf{W}(\mathbf{D}-$ I) $\mathbf{X}^{-1}$. Left multiplication by $\mathbf{X}$ and right multiplication by $\mathbf{X}^{-1}$ of both sides of (43) yields

$$
\begin{aligned}
\mathbf{I}= & \mu(\mathbf{X D} \mathbf{W}+\mathbf{X}) \boldsymbol{\Phi}_{\overline{\mathbf{s}}}^{H} \overline{\mathbf{S}} \boldsymbol{\Phi}_{\overline{\mathbf{s}}}\left(\mathbf{W}(\mathbf{D}-\mathbf{I}) \mathbf{X}^{-1}+\mathbf{X}^{-1}\right) \\
= & \mu(\mathbf{X D} \mathbf{W}+\mathbf{X}) \boldsymbol{\Phi}_{\overline{\mathbf{s}}}^{H} \overline{\mathbf{S}} \boldsymbol{\Phi}_{\overline{\mathbf{s}}}\left(\mathbf{W} \mathbf{D} \mathbf{X}+\mathbf{X}^{-1}\right) \\
= & \mu\left(\mathbf{X D} \mathbf{W} \mathbf{D X}+\mathbf{X}^{2} \mathbf{D}\right) \mathbf{X}^{-1} \mathbf{D}^{-1} \boldsymbol{\Phi}_{\overline{\mathbf{s}}}^{H} \overline{\mathbf{S}} \boldsymbol{\Phi}_{\overline{\mathbf{s}}} \mathbf{D}^{-1} \mathbf{X}^{-1} \\
& \times(\mathbf{X D} \mathbf{W} \mathbf{D X}+\mathbf{D}) \\
= & \mu(\mathbf{X D} \mathbf{W} \mathbf{D X}+(\mathbf{D}-\mathbf{I})) \mathbf{X}^{-1} \mathbf{D}^{-1} \boldsymbol{\Phi}_{\overline{\mathbf{s}}}^{H} \overline{\mathbf{S}} \boldsymbol{\Phi}_{\overline{\mathbf{s}}} \mathbf{D}^{-1} \mathbf{X}^{-1} \\
& \times(\mathbf{X D} \mathbf{W} \mathbf{D X}+\mathbf{D}) .
\end{aligned}
$$

Equation (44) depends on $\mathbf{Q}^{*}$ through the term XD W DX, where we recall (42). Again using the fact that $\mathbf{D X}=(\mathbf{D}-$ I) $\mathbf{X}^{-1}$ in the last equation, one can easily show that

$$
\begin{array}{r}
\mathbf{I}=\mu(\mathbf{X D} W \mathbf{D X}+\mathbf{D}) \mathbf{X}^{-1} \mathbf{D}^{-1} \boldsymbol{\Phi}_{\overline{\mathbf{s}}}^{H} \overline{\mathbf{S}} \boldsymbol{\Phi}_{\overline{\mathbf{s}}} \mathbf{D}^{-1} \mathbf{X}^{-1} \\
\times(\mathbf{X} \mathbf{D} \mathbf{W} \mathbf{D} \mathbf{X}+(\mathbf{D}-\mathbf{I})) .
\end{array}
$$

\section{Define $\mathbf{K}$ as}

$$
\mathbf{K}=\mathbf{X} \mathbf{D} \mathbf{W} \mathbf{D X}=\left(\mathbf{I}-\mathbf{D}^{-1}\right)^{\frac{1}{2}} \mathbf{D} \mathbf{W} \mathbf{D}\left(\mathbf{I}-\mathbf{D}^{-1}\right)^{\frac{1}{2}}
$$

where $\mathbf{D}$ and $\mathbf{W}$ are respectively given by (38) and (42). Moreover, let

$$
\begin{aligned}
\widetilde{\mathbf{S}} & =\mathbf{X}^{-1} \mathbf{D}^{-1} \boldsymbol{\Phi}_{\overline{\mathbf{s}}}^{H} \overline{\mathbf{S}} \boldsymbol{\Phi}_{\overline{\mathbf{s}}} \mathbf{D}^{-1} \mathbf{X}^{-1} \\
& =\left(\mathbf{I}-\mathbf{D}^{-1}\right)^{-\frac{1}{2}} \mathbf{D}^{-1} \boldsymbol{\Phi}_{\overline{\mathbf{s}}}^{H} \overline{\mathbf{S}} \boldsymbol{\Phi}_{\overline{\mathbf{s}}} \mathbf{D}^{-1}\left(\mathbf{I}-\mathbf{D}^{-1}\right)^{-\frac{1}{2}} .
\end{aligned}
$$

Using (46) and (47) in (44) and (45), we have

$$
\begin{aligned}
\mathbf{I} & =\mu(\mathbf{K}+\mathbf{D}) \widetilde{\mathbf{S}}(\mathbf{K}+(\mathbf{D}-\mathbf{I})) \\
& =\mu(\mathbf{K}+(\mathbf{D}-\mathbf{I})) \widetilde{\mathbf{S}}(\mathbf{K}+\mathbf{D}) .
\end{aligned}
$$

We note from (48) that

$$
\begin{aligned}
\mu \widetilde{\mathbf{S}} & =(\mathbf{K}+\mathbf{D})^{-1}(\mathbf{K}+(\mathbf{D}-\mathbf{I}))^{-1} \\
& =(\mathbf{K}+(\mathbf{D}-\mathbf{I}))^{-1}(\mathbf{K}+\mathbf{D})^{-1} .
\end{aligned}
$$

This result implies that, for the optimal $\mathbf{Q}^{*}$, matrices $(\mathbf{K}+(\mathbf{D}-$ $\mathbf{I}))^{-1},(\mathbf{K}+\mathbf{D})^{-1}$ and $\widetilde{\mathbf{S}}$ commute and have the same eigenvectors [16, Theorem 1.3.12, Corollary 4.5.18].

Theorem 2: The optimal full-rank input covariance matrix that attains the secrecy capacity for the average power constraint is given by

$$
\mathbf{Q}^{*}=\overline{\mathbf{S}}^{\frac{1}{2}} \boldsymbol{\Phi}_{\overline{\mathbf{s}}}\left(\mathbf{I}-\mathbf{D}^{-1}\right)^{-\frac{1}{2}} \mathbf{D}^{-1} \mathbf{K} \mathbf{D}^{-1}\left(\mathbf{I}-\mathbf{D}^{-1}\right)^{-\frac{1}{2}} \boldsymbol{\Phi}_{\overline{\mathbf{S}}}^{H} \overline{\mathbf{S}}^{\frac{1}{2}}
$$

iff

- $\mathbf{H}^{H} \mathbf{H}-\mathbf{G}^{H} \mathbf{G} \succ \mathbf{0}$, and

- for the given $P_{t}$,

$$
\mathbf{K} \succ \mathbf{0}
$$

where $\boldsymbol{\Phi}_{\overline{\mathbf{s}}}$ and $\mathbf{D}$ are given by (38), $\mathbf{K}$ is defined by (46), and $\overline{\mathbf{S}}=\left(\mathbf{H}^{H} \mathbf{H}-\mathbf{G}^{H} \mathbf{G}\right)^{-1}$.

Proof: We have previously shown that (36), (37) result in (49), where the dependence on $\mathbf{Q}^{*}$ is through $\mathbf{K}$ in (46). Denote the eigenvalue decomposition of $\widetilde{\mathbf{S}}$ in (47) and (49) as

$$
\widetilde{\mathbf{S}}=\mathbf{U} \boldsymbol{\Omega} \mathbf{U}^{H},
$$

where $\mathbf{U}$ is unitary and $\boldsymbol{\Omega}$ is diagonal. Based on the argument made after (49), we have

$$
\begin{aligned}
\mathbf{K}+(\mathbf{D}-\mathbf{I}) & =\mathbf{U} \boldsymbol{\Sigma} \mathbf{U}^{H} \\
\mathbf{K}+\mathbf{D} & =\mathbf{U} \boldsymbol{\Delta} \mathbf{U}^{H},
\end{aligned}
$$

where $\boldsymbol{\Sigma}$ and $\boldsymbol{\Delta}$ are diagonal, and $\boldsymbol{\Delta}=\boldsymbol{\Sigma}+\mathbf{I}$. Substituting (52) and (53) in (48), and noting that $\mathbf{U}^{H} \mathbf{U}=\mathbf{U} \mathbf{U}^{H}=\mathbf{I}$, and that $\boldsymbol{\Sigma}, \boldsymbol{\Delta}$ and $\boldsymbol{\Omega}$ are all diagonal, we have

$$
\mathbf{I}=\mu \mathbf{U} \boldsymbol{\Sigma} \boldsymbol{\Omega} \boldsymbol{\Delta} \mathbf{U}^{H}=\mu \boldsymbol{\Sigma} \boldsymbol{\Omega} \boldsymbol{\Delta}=\mu\left(\boldsymbol{\Sigma}^{2}+\boldsymbol{\Sigma}\right) \boldsymbol{\Omega} .
$$

From (54), we can solve for the diagonal matrix $\boldsymbol{\Sigma}$, based on the known diagonal matrix $\boldsymbol{\Omega}$, and obtain

$$
\boldsymbol{\Sigma}=\frac{1}{2}\left(-\mathbf{I}+\left(\mathbf{I}+\frac{4}{\mu} \boldsymbol{\Omega}^{-1}\right)^{\frac{1}{2}}\right) .
$$

Using (55) in (53), we have

$$
\mathbf{K}=\left(\mathbf{U} \boldsymbol{\Sigma} \mathbf{U}^{H}+\mathbf{I}-\mathbf{D}\right) .
$$

Substituting $\mathbf{K}$ back into (46) and (42), a straightforward computation yields $\mathrm{Q}^{*}$ given by (50).

It should be noted that the only unknown parameter in (50) is the scalar $\mu>0$, which appears in the diagonal matrix $\boldsymbol{\Sigma}$, as shown in (55). The Lagrange multiplier $\mu$ is chosen to satisfy the power constraint $\operatorname{Tr}\left(\mathbf{Q}^{*}\right)=P_{t}$. As (55) shows, $\operatorname{Tr}\left(\mathbf{Q}^{*}\right)$ is monotonically decreasing with $\mu$ :

$$
\begin{aligned}
\lim _{\mu \rightarrow 0} \operatorname{Tr}\left(\mathbf{Q}^{*}\right) & =\infty \text { and } \lim _{\mu \rightarrow \infty} \operatorname{Tr}\left(\mathbf{Q}^{*}\right) \\
& =-\operatorname{Tr}\left(\overline{\mathbf{S}}^{\frac{1}{2}} \boldsymbol{\Phi}_{\overline{\mathbf{s}}} \mathbf{D}^{-1} \boldsymbol{\Phi}_{\overline{\mathbf{s}}}^{H} \overline{\mathbf{S}}^{\frac{1}{2}}\right)<0 .
\end{aligned}
$$

Thus for any transmit power $P_{t}$, there exists a Lagrange multiplier $\mu>0$ for which $\operatorname{Tr}\left(\mathbf{Q}^{*}\right)=P_{t}$. The appropriate value of $\mu$ can easily be found using, for example, the bisection method.

Finally, one can easily confirm that $\mathbf{Q}^{*}$ in (50) is a valid solution, i.e. that $\mathbf{Q}^{*} \succ \mathbf{0}$, if $\mathbf{H}^{H} \mathbf{H}-\mathbf{G}^{H} \mathbf{G} \succ \mathbf{0}$ and (51) is satisfied for the given $P_{t}$.

The flowchart in Fig. 1 summarizes the steps required to calculate the optimal full-rank $\mathbf{Q}^{*}$ when $\mathbf{H}^{H} \mathbf{H}-\mathbf{G}^{H} \mathbf{G} \succ \mathbf{0}$. It is interesting to note that only the term $\boldsymbol{\Sigma}$ in Step 5 depends on the channel matrices $(\mathbf{H}, \mathbf{G})$, and also the average power constraint $P_{t}$; all other terms used to form $\mathbf{Q}^{*}$ depend only on the channel matrices $(\mathbf{H}, \mathbf{G})$. 


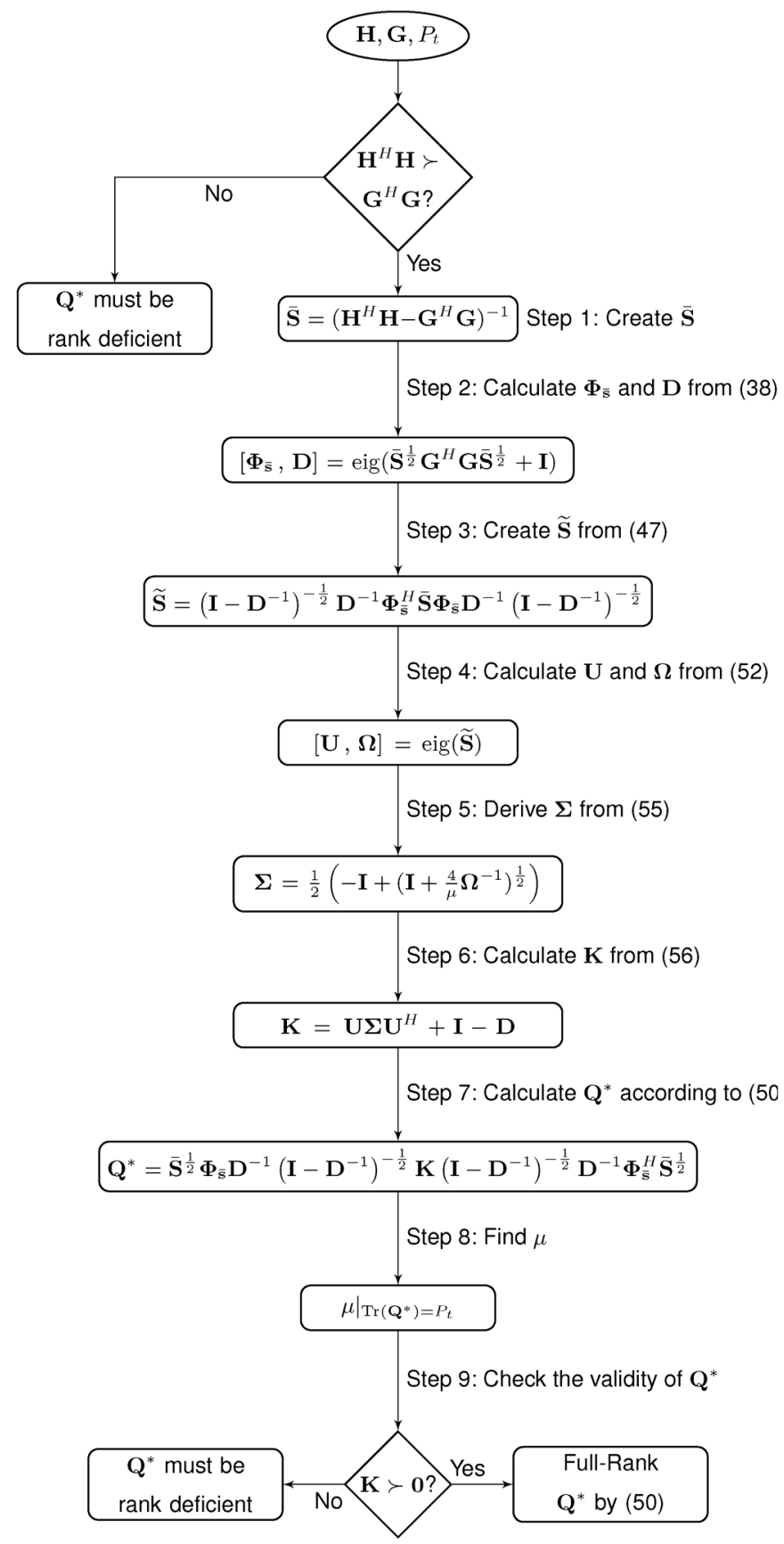

Fig. 1. Flowchart for obtaining the optimal full-rank $\mathbf{Q}^{*}$.

From (39) and (50), and recalling that $\boldsymbol{\Phi}_{\overline{\mathbf{s}}}$ in (39) is unitary we have:

$$
\begin{aligned}
& \log \left|\mathbf{I}+\mathbf{H Q}^{*} \mathbf{H}^{H}\right| \\
& =\log \left|\mathbf{I}+\mathbf{H}^{H} \mathbf{H} \mathbf{Q}^{*}\right| \\
& =\log \mid \mathbf{I}+\mathbf{D}\left(\mathbf{I}-\mathbf{D}^{-1}\right)^{-\frac{1}{2}} \mathbf{D}^{-1}\left(\mathbf{U} \boldsymbol{\Sigma} \mathbf{U}^{H}+\mathbf{I}-\mathbf{D}\right) \mathbf{D}^{-1} \\
& \quad \quad \times\left(\mathbf{I}-\mathbf{D}^{-1}\right)^{-\frac{1}{2}} \mid \\
& =\log \left|\mathbf{I}+\left(\mathbf{U} \boldsymbol{\Sigma} \mathbf{U}^{H}+\mathbf{I}-\mathbf{D}\right) \mathbf{D}^{-1}\left(\mathbf{I}-\mathbf{D}^{-1}\right)^{-1}\right| \\
& =\log \left|\mathbf{I}+\left(\mathbf{U} \boldsymbol{\Sigma} \mathbf{U}^{H}+\mathbf{I}-\mathbf{D}\right)(\mathbf{D}-\mathbf{I})^{-1}\right| \\
& =\log \left|\mathbf{U} \mathbf{\Sigma} \mathbf{U}^{H}(\mathbf{D}-\mathbf{I})^{-1}\right|=\log \left|\mathbf{\Sigma}(\mathbf{D}-\mathbf{I})^{-1}\right| \\
& =\log |\mathbf{\Sigma}|-\log |\mathbf{D}-\mathbf{I}|
\end{aligned}
$$

where (57) comes from the fact that $\left|\mathbf{I}+\mathbf{A}^{B}\right|=|\mathbf{I}+\mathbf{B A}|$, and (58) holds because $\mathbf{U}$ is unitary. Similarly, one can show that

$$
\log \left|\mathbf{I}+\mathbf{G Q}^{*} \mathbf{G}^{H}\right|=\log |\boldsymbol{\Sigma}+\mathbf{I}|-\log |\mathbf{D}| .
$$

Remark 4: By substituting (58) and (59) into (28), the optimal input covariance matrix $\mathbf{Q}^{*}$ given by Theorem 2 attains the secrecy capacity

$$
\begin{aligned}
\mathcal{C}_{\text {sec }}\left(P_{t}\right) & =\log |\boldsymbol{\Sigma}|-\log |\boldsymbol{\Sigma}+\mathbf{I}|+\log |\mathbf{D}|-\log |\mathbf{D}-\mathbf{I}| \\
& =\log \left|\mathbf{I}-(\boldsymbol{\Sigma}+\mathbf{I})^{-1}\right|+\log \left|\mathbf{I}+(\mathbf{D}-\mathbf{I})^{-1}\right|,
\end{aligned}
$$

where $\boldsymbol{\Sigma}$ and $\mathbf{D}$ are diagonal matrices, respectively given by (55) and (38). Note that while both log terms in (60) return non-negative values, the first term depends on both the channels and the power $P_{t}$, while the second term depends only on the channels (see Lemma 5).

\section{B. Limiting Cases for the Full-Rank Solution}

This section discusses some interesting points regarding the optimal solution in (50). For the following observations, one can assume when required that both conditions for a full-rank $\mathbf{Q}^{*}$ given in Theorem (2) are satisfied. Let $\gamma_{i}, i=1, \cdots, n_{t}$, be the generalized eigenvalues of the pencil $\left(\mathbf{H}^{H} \mathbf{H}, \mathbf{G}^{H} \mathbf{G}\right)$. Then from [17], $\gamma_{i}=\sigma_{i}^{2}$, where $\sigma_{i}$ is the $i$ th generalized singular value of $(\mathbf{H}, \mathbf{G})$.

Lemma 5: The second term in the secrecy capacity expression (60) depends only on $\mathbf{H}, \mathbf{G}$ and is equal to $\sum_{i=1}^{n_{t}} \log \left(\sigma_{i}^{2}\right)$.

Proof: From (39) we have:

$$
\begin{aligned}
\mathbf{H}^{H} \mathbf{H} \overline{\mathbf{S}}^{\frac{1}{2}} \boldsymbol{\Phi}_{\overline{\mathbf{s}}} & =\overline{\mathbf{S}}^{-\frac{1}{2}} \boldsymbol{\Phi}_{\overline{\mathbf{s}}} \mathbf{D} \\
& =\mathbf{G}^{H} \mathbf{G} \overline{\mathbf{S}}^{\frac{1}{2}} \boldsymbol{\Phi}_{\overline{\mathbf{s}}}(\mathbf{D}-\mathbf{I})^{-1} \mathbf{D} \\
& =\mathbf{G}^{H} \mathbf{G} \overline{\mathbf{S}}^{\frac{1}{2}} \boldsymbol{\Phi}_{\overline{\mathbf{s}}}\left(\mathbf{I}+(\mathbf{D}-\mathbf{I})^{-1}\right),
\end{aligned}
$$

where (61) comes from (40). Thus, from [16], the generalized eigenvalue matrix of $\left(\mathbf{H}^{H} \mathbf{H}, \mathbf{G}^{H} \mathbf{G}\right)$ is $\left(\mathbf{I}+(\mathbf{D}-\mathbf{I})^{-1}\right)$, which completes the proof. We have

$$
\log \left|\mathbf{I}+(\mathbf{D}-\mathbf{I})^{-1}\right|=\sum_{i=1}^{n_{t}} \log \left(\sigma_{i}^{2}\right)
$$

where

$$
\sigma_{i}^{2}=1+\frac{1}{d_{i}-1}
$$

and $d_{i}$ is the $i$ th diagonal element of the positive diagonal matrix D.

Note that the definition of generalized singular values here is slightly different from that in [4]. Here $\sigma_{i}$ may be $\infty$, while this is not the case in [4]. More precisely, for the case of $\operatorname{rank}(\mathbf{G})=$ $n_{e}<n_{t}$, from (40) we have that $n_{t}-n_{e}$ diagonal elements of $\mathbf{D}$ are equal to one. From (62), the $\sigma_{i}$ corresponding to $d_{i}=1$ tend to $\infty$.

Lemma 6: In the high SNR scenario $\left(P_{t} \rightarrow \infty\right)$ and for the case of $\operatorname{rank}(\mathbf{G})=n_{t}$, the asymptotic form of the exact secrecy capacity (60) is simply given by

$$
\mathcal{C}_{\text {sec }}=\log \left|\mathbf{I}+(\mathbf{D}-\mathbf{I})^{-1}\right|=\sum_{i=1}^{n_{t}} \log \left(\sigma_{i}^{2}\right),
$$

as originally derived in [4, Theorem 2]. 
Proof: For $P_{t} \rightarrow \infty$, the Lagrange parameter satisfies $\mu \rightarrow 0$, as mentioned after Theorem 2 . Also it should be noted that, for the case of $\operatorname{rank}(\mathbf{G})=n_{t}$, the matrix $\widetilde{\mathbf{S}}$, which is independent of $P_{t}$ and is given by (47), will have finite-valued eigenvalues. Thus as $\mu \rightarrow 0$, the diagonal elements of $\boldsymbol{\Sigma}$, given by (55), go to $\infty$. Consequently, the first log term in (60) disappears as $P_{t} \rightarrow \infty$.

It is also interesting to consider the optimal solution in (50) for the case that the eavesdropper's channel is very weak, e.g. $\mathbf{G} \rightarrow \mathbf{0}$. For this specific case, the wiretap channel simplifies to a point-to-point MIMO Gaussian link, where the optimal input covariance matrix under the average power constraint is known to be $\boldsymbol{\Phi}_{H}\left(\frac{1}{\mu} \mathbf{I}-\boldsymbol{\Lambda}_{H}^{-1}\right)^{+} \boldsymbol{\Phi}_{H}^{H}$, and is found via the standard water-filling solution, where unitary $\boldsymbol{\Phi}_{H}$ and diagonal $\boldsymbol{\Lambda}_{H}$ are obtained from the eigenvalue decomposition $\mathbf{H}^{H} \mathbf{H}=\boldsymbol{\Phi}_{H} \boldsymbol{\Lambda}_{H} \boldsymbol{\Phi}_{H}^{H}$.

Lemma 7: The optimal solution in (50) converges to the conventional water-filling solution as $\mathbf{G} \rightarrow \mathbf{0}$ :

$$
\lim _{\mathbf{G} \rightarrow \mathbf{0}} \mathbf{Q}^{*}=\boldsymbol{\Phi}_{H}\left(\mu^{-1} \mathbf{I}-\boldsymbol{\Lambda}_{H}^{-1}\right)^{+} \boldsymbol{\Phi}_{H}^{H} .
$$

Proof: Using (39) and via simple calculations, we note that for any $\mathbf{G},(50)$ can be rewritten as

$$
\begin{aligned}
\mathbf{Q}^{*}=\overline{\mathbf{S}}^{\frac{1}{2}} \boldsymbol{\Phi}_{\overline{\mathbf{S}}}(\mathbf{I}- & \left.\mathbf{D}^{-1}\right)^{-\frac{1}{2}} \mathbf{D}^{-1} \mathbf{U} \boldsymbol{\Sigma} \mathbf{U}^{H} \mathbf{D}^{-1} \\
& \times\left(\mathbf{I}-\mathbf{D}^{-1}\right)^{-\frac{1}{2}} \boldsymbol{\Phi}_{\overline{\mathbf{S}}}^{H} \overline{\mathbf{S}}^{\frac{1}{2}}-\left(\mathbf{H}^{H} \mathbf{H}\right)^{-1} .
\end{aligned}
$$

From (40), we note that when $\mathbf{G} \rightarrow \mathbf{0}$ then $\mathbf{D} \rightarrow \mathbf{I}$. Next from (39), $\boldsymbol{\Phi}_{\overline{\mathbf{s}}} \rightarrow \boldsymbol{\Phi}_{H}$. Also note that $\overline{\mathbf{S}}=\left(\mathbf{H}^{H} \mathbf{H}-\mathbf{G}^{H} \mathbf{G}\right)^{-1} \rightarrow$ $\left(\mathbf{H}^{H} \mathbf{H}\right)^{-1}$ when $\mathbf{G} \rightarrow \mathbf{0}$. Using these facts in (47), and after some straightforward calculations, we have $\widetilde{\mathbf{S}} \rightarrow(\mathbf{D}-\mathbf{I})^{-1} \mathbf{D}^{-1} \boldsymbol{\Lambda}_{H}^{-1}=\boldsymbol{\Omega}$, and $\mathbf{U} \rightarrow \mathbf{I}$ in (52). Using these in (65), we have

$$
\begin{aligned}
\mathbf{Q}^{*} \rightarrow & \boldsymbol{\Phi}_{H}(\mathbf{D}-\mathbf{I})^{-1} \mathbf{D}^{-1} \boldsymbol{\Lambda}_{H}^{-1} \boldsymbol{\Sigma} \boldsymbol{\Phi}_{H}^{H}-\left(\mathbf{H}^{H} \mathbf{H}\right)^{-1} \\
\rightarrow & \frac{1}{2} \boldsymbol{\Phi}_{H}(\mathbf{D}-\mathbf{I})^{-1} \boldsymbol{\Lambda}_{H}^{-1}\left(-\mathbf{I}+\left(\mathbf{I}+\frac{4}{\mu}(\mathbf{D}-\mathbf{I}) \boldsymbol{\Lambda}_{H}\right)^{\frac{1}{2}}\right) \\
& \times \boldsymbol{\Phi}_{H}^{H}-\left(\mathbf{H}^{H} \mathbf{H}\right)^{-1} \\
\rightarrow & \frac{1}{\mu} \mathbf{I}-\left(\mathbf{H}^{H} \mathbf{H}\right)^{-1}=\boldsymbol{\Phi}_{H}\left(\mu^{-1} \mathbf{I}-\boldsymbol{\Lambda}_{H}^{-1}\right)^{+} \boldsymbol{\Phi}_{H}^{H}
\end{aligned}
$$

where in obtaining (66) we used the fact that $\boldsymbol{\Lambda}_{H}$ and $\mathbf{D}$ are diagonal, and $\mathbf{D} \rightarrow \mathbf{I}$ when $\mathbf{G} \rightarrow \mathbf{0}$.

\section{Rank Deficient Solution}

It is important to note that Theorem 2 reveals the necessary and sufficient conditions for a full rank optimal $\mathbf{Q}^{*}$. More precisely, if $\mathbf{H}^{H} \mathbf{H}-\mathbf{G}^{H} \mathbf{G}$ is not positive definite, or the matrix inequality in (51) does not hold, then for the given wiretap channel with parameters $\left(\mathbf{H}, \mathbf{G}, P_{t}\right)$, the optimal input covariance matrix $\mathbf{Q}^{*}$ is not full-rank and the expression in (50) cannot be used. In the following, we characterize some properties of the optimal input covariance in the rank-deficient case, and we propose an algorithm to find $\mathbf{Q}^{*}$ using a search over a smaller space than that required in (13).

Theorem 3: For a general MIMO Gaussian wiretap channel $\left(\mathbf{H}, \mathbf{G}, P_{t}\right)$ with a rank deficient optimal input covariance matrix $\mathbf{Q}^{*}$, there exists an equivalent wiretap channel $\left(\mathbf{H}_{e q}, \mathbf{G}_{e q}, P_{t}\right)$, such that $\mathcal{C}_{s e c}\left(\mathbf{H}, \mathbf{G}, P_{t}\right)=$ $\mathcal{C}_{s e c}\left(\mathbf{H}_{e q}, \mathbf{G}_{e q}, P_{t}\right)$ and the optimal input covariance matrix $\mathbf{Q}_{e q}^{*}$ for the equivalent channel is full-rank.

Proof: Let $\widehat{b}=\operatorname{rank}\left(\mathbf{Q}^{*}\right)$. The proof is found in Appendix C. In particular, it is shown that $\mathbf{Q}^{*}=$ $\mathbf{U}_{c} \mathbf{Q}_{e q}^{*} \mathbf{U}_{c}^{H}$, where $\mathbf{U}_{c} \in \mathbb{C}^{n_{t} \times \widehat{b}}$ satisfies $\mathbf{U}_{c}^{H} \mathbf{U}_{c}=\mathbf{I}$ and $\mathbf{H}_{e q}^{H} \mathbf{H}_{e q}-\mathbf{G}_{e q}^{H} \mathbf{G}_{e q} \succ \mathbf{0}$, where $\mathbf{H}_{e q}=\mathbf{H} \mathbf{U}_{c}$ and $\mathbf{G}_{e q}=\mathbf{G U}_{c}$.

Based on Theorem 3, the following algorithm can, at least conceptually, be used to obtain the optimal transmit covariance for any general MIMO Gaussian wiretap channel with $\left(\mathbf{H}, \mathbf{G}, P_{t}\right)$, excluding the special case $\mathbf{H}^{H} \mathbf{H}-\mathbf{G}^{H} \mathbf{G} \preceq \mathbf{0}$ for which the secrecy capacity is 0 , as mentioned in Remark 1. (In the discussion below, recall that $m$ represents number of positive eigenvalues of $\mathbf{H}^{H} \mathbf{H}-\mathbf{G}^{H} \mathbf{G}$, and is equal to the maximum possible value for $\operatorname{rank}\left(\mathbf{Q}^{*}\right)$, as mentioned in Theorem 1.)

1) Apply Theorem 2. If both conditions in Theorem 2 are satisfied, then $\mathbf{Q}^{*}$ is given by $(50)$ and $\mathcal{C}_{\text {sec }}\left(\mathbf{H}, \mathbf{G}, P_{t}\right)$ is given by (60).

2) If Theorem 2 determines that $\mathbf{Q}^{*}$ is not full-rank, the secrecy capacity is found through a search over the set $\mathcal{U}=$ $\left\{\mathbf{U}_{b} \in \mathbb{C}^{n_{t} \times b}: \mathbf{U}_{b}^{H}\left(\mathbf{H}^{H} \mathbf{H}-\mathbf{G}^{H} \mathbf{G}\right) \mathbf{U}_{b} \succ \mathbf{0}, \mathbf{U}_{b}^{H} \mathbf{U}_{b}=\right.$ $\mathbf{I}, \mathbf{Q}_{b}^{*} \succ \mathbf{0}$ for $\left.\left(\mathbf{H}_{b}, \mathbf{G}_{b}, P_{t}\right), b=1, \ldots, m\right\}$ :

$$
\mathcal{C}_{\text {sec }}\left(\mathbf{H}, \mathbf{G}, P_{t}\right)=\max _{\mathbf{U}_{b} \in \mathcal{U}} \mathcal{C}_{\text {sec }}\left(\mathbf{H}_{b}, \mathbf{G}_{b}, P_{t}\right),
$$

where for each $\mathbf{U}_{b} \in \mathcal{U}$, we define $\mathbf{H}_{b}=\mathbf{H U}_{b}$, $\mathbf{G}_{b}=\mathbf{G U}_{b}$, and $\mathbf{H}_{b}^{H} \mathbf{H}_{b}-\mathbf{G}_{b}^{H} \mathbf{G}_{b} \succ \mathbf{0}$. Also, $\mathcal{C}_{\text {sec }}\left(\mathbf{H}_{b}, \mathbf{G}_{b}, P_{t}\right)$ is obtained from (60), after applying Theorem 2 to the wiretap channel $\left(\mathbf{H}_{b}, \mathbf{G}_{b}, P_{t}\right) . \mathbf{Q}_{b}^{*}$ is the optimal full-rank solution for the channel $\left(\mathbf{H}_{b}, \mathbf{G}_{b}, P_{t}\right)$, which attains $\mathcal{C}_{\text {sec }}\left(\mathbf{H}_{b}, \mathbf{G}_{b}, P_{t}\right)$ and is given by (50).

3) The optimal input covariance matrix $Q^{*}$ that attains $\mathcal{C}_{\text {sec }}\left(\mathbf{H}, \mathbf{G}, P_{t}\right)$ is given by $\mathbf{Q}^{*}=\mathbf{U}_{c} \mathbf{Q}_{e q}^{*} \mathbf{U}_{c}^{H}$, where

$$
\mathbf{U}_{c}=\arg \max _{\mathbf{U}_{b} \in \mathcal{U}} \mathcal{C}_{s e c}\left(\mathbf{H}_{b}, \mathbf{G}_{b}, P_{t}\right),
$$

and $\mathbf{Q}_{e q}^{*}$ is obtained from (50) by applying Theorem 2 to the wiretap channel $\left(\mathbf{H}_{e q}, \mathbf{G}_{e q}, P_{t}\right)$, where $\mathbf{H}_{e q}=\mathbf{H U}_{c}$ and $\mathbf{G}_{e q}=\mathbf{G U}_{c}$.

Comparing problems (13) and (67), we note that the above algorithm considerably restricts the search space. We can restrict the search space even more by noting that for the case of $\operatorname{rank}\left(\mathbf{Q}^{*}\right)=1$, it is shown in [11] that the optimal input covariance matrix is given by $\mathbf{Q}^{*}=P_{t} \mathbf{u} \mathbf{u}^{H}$, where $\mathbf{u}$ is the normalized principal eigenvector corresponding to the largest eigenvalue $\lambda_{1}$ of the pencil $\left(\mathbf{I}+P_{t} \mathbf{H}^{H} \mathbf{H}, \mathbf{I}+P_{t} \mathbf{G}^{H} \mathbf{G}\right)$. For this case, if $\lambda_{1}>1$, the secrecy capacity is $\mathcal{C}_{\text {sec }}\left(\mathbf{H}, \mathbf{G}, P_{t}\right)=\log \left(\lambda_{1}\right)$. Consequently, problem (67) can be written as

$$
\mathcal{C}_{\text {sec }}\left(\mathbf{H}, \mathbf{G}, P_{t}\right)=\max \left(\max _{\mathbf{U}_{b} \in \mathcal{U}^{\prime}} \mathcal{C}_{s e c}\left(\mathbf{H}_{b}, \mathbf{G}_{b}, P_{t}\right), \log \left(\lambda_{1}\right)\right),
$$

where one need only consider $\mathbf{U}_{b}$ of rank 2 or higher, and hence $\mathcal{U}^{\prime}=\left\{\mathbf{U}_{b} \in \mathbb{C}^{n_{t} \times b}: \mathbf{U}_{b}^{H}\left(\mathbf{H}^{H} \mathbf{H}-\mathbf{G}^{H} \mathbf{G}\right) \mathbf{U}_{b} \succ \mathbf{0}, \mathbf{U}_{b}^{H} \mathbf{U}_{b}=\right.$ $\mathbf{I}, \mathbf{Q}_{b}^{*} \succ \mathbf{0}$ for $\left.\left(\mathbf{H}_{b}, \mathbf{G}_{b}, P_{t}\right), b=2, \ldots, m\right\}$. 


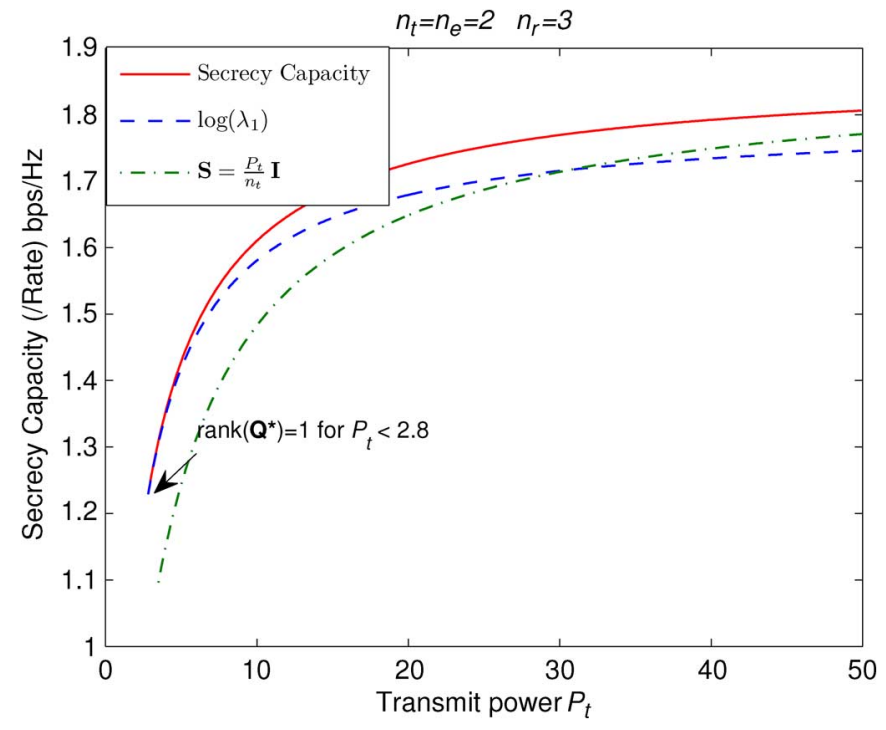

Fig. 2. Secrecy Capacity versus $P_{t}$ for $n_{t}=n_{e}=2$ and $n_{r}=3$. Solid curve represents secrecy capacity and dotted curve indicates the achievable secrecy rate using a rank-one input covariance matrix.

\section{Numerical Results}

In the first example, we consider a MIMO wiretap channel with $n_{t}=n_{e}=2, n_{r}=3$ and channel matrices given by

$$
\begin{aligned}
& \mathbf{H}=\left[\begin{array}{cc}
0.32-0.52 i & 0.83+1.15 i \\
0.51-0.26 i & 0.06-0.15 i \\
-0.11+0.81 i & 0.29+0.68 i
\end{array}\right] \\
& \mathbf{G}=\left[\begin{array}{cc}
0.03-0.70 i & -0.32-0.32 i \\
0.24-0.11 i & 1.36+0.18 i
\end{array}\right],
\end{aligned}
$$

which satisfy $\mathbf{H}^{H} \mathbf{H}-\mathbf{G}^{H} \mathbf{G} \succ \mathbf{0}$. Fig. 2 shows the secrecy capacity as a function of transmit power $P_{t}$. Note that in this example, the optimal $\mathrm{Q}^{*}$ is not full-rank for $P_{t}<2.8$. For comparison, the figure also depicts the achievable secrecy rate using a rank-one (beamforming) covariance $\mathbf{Q}=P_{t} \mathbf{u u}{ }^{H}$, which results to $R_{\text {sec }}=\log \lambda_{1}$, as shown in [4]-[11]. Also shown is the secrecy capacity achieved with a matrix constraint corresponding to an equal power allocation across the transmit antennas: $\mathbf{S}=$ $\frac{P_{t}}{n_{t}} \mathbf{I}$. As expected, the secrecy capacity achieved by the average power constraint is considerably larger, although the gap narrows as $P_{t}$ increases since in this example $\mathbf{G}^{H} \mathbf{G}$ is full-rank (i.e., there is no null-space in the eavesdropper's channel).

In Fig. 3 we consider another case where $\mathbf{H}^{H} \mathbf{H}-\mathbf{G}^{H} \mathbf{G} \succ \mathbf{0}$, here with $n_{t}=n_{r}=3, n_{e}=2$ and channel matrices given by

$$
\begin{aligned}
\mathbf{H} & =\left[\begin{array}{ccc}
0.24-0.83 i & 1.32+0.33 i & 0.94+0.58 i \\
-0.22-1.69 i & -0.12+0.60 i & -0.6-0.22 i \\
0.36-0.82 i & 1.35-0.23 i & -1.03-0.32 i
\end{array}\right] \\
\mathbf{G} & =\left[\begin{array}{ccc}
0.36+0.04 i & 0.70+0.75 i & 0.07-0.07 i \\
-0.44+0.10 i & -0.72+0.15 i & -0.61+0.45 i
\end{array}\right] .
\end{aligned}
$$

For this example, the optimal $\mathrm{Q}^{*}$ is only full-rank for $P_{t}>$ 0.75 . It is interesting to note that in this example, where $\mathbf{G}^{H} \mathbf{G}$ is not full rank and hence there exists a non-trivial null space in $\mathbf{G}$, as $P_{t}$ increases the difference between the optimal solution with an average power constraint and the solution under the matrix constraint $\mathbf{S}=\frac{P_{t}}{n_{t}} \mathbf{I}$ increases.

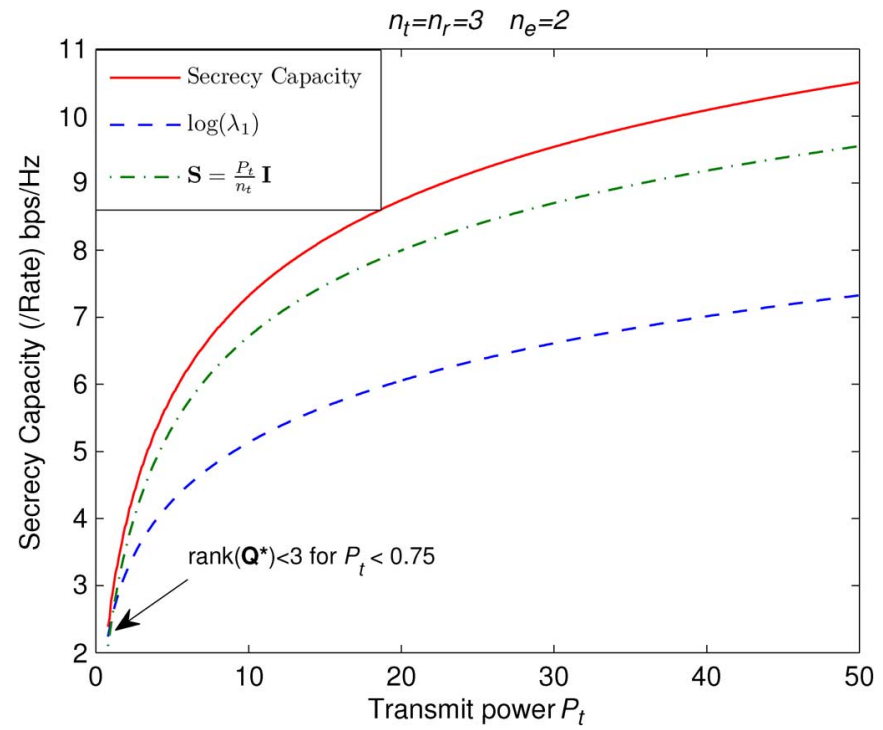

Fig. 3. Secrecy Capacity versus $P_{t}$ for $n_{t}=n_{r}=3$ and $n_{e}=2$. Solid curve represents secrecy capacity and dotted curve indicates the achievable secrecy rate using a rank-one input covariance matrix.

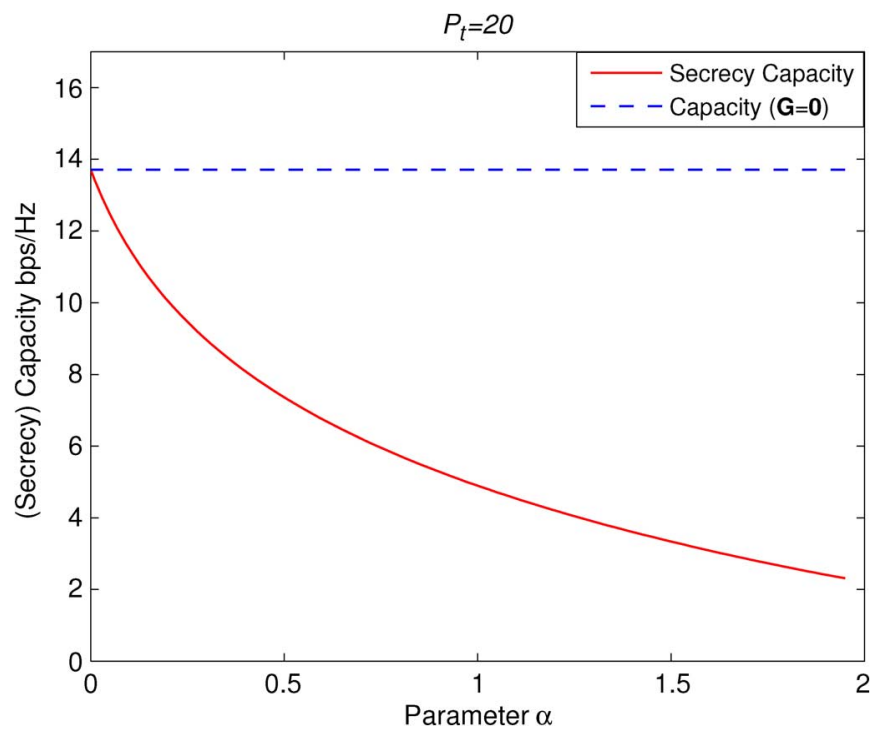

Fig. 4. Secrecy Capacity versus $\alpha$ for $P_{t}=20$. Solid curve represents secrecy capacity and dashed curve indicates the point to point capacity.

Finally in Fig. 4, we compare the standard point-to-point capacity without secrecy constraints to the secrecy capacity given by (60). In this example, $P_{t}=20$, direct channel $\mathbf{H}$ is given by

$$
\mathbf{H}=\left[\begin{array}{ccc}
0.89+0.54 i & -0.06+0.60 i & 0.48-1.11 i \\
0.46 & -0.44+0.80 i & -1.07+0.63 i \\
1.40-0.13 i & 0.17-0.82 i & 0.59-0.31 i \\
0.43-0.23 i & 0.03+1.35 i & 0.44-0.07 i
\end{array}\right]
$$

but the cross channel $\mathbf{G}$ is assumed to satisfy $\mathbf{G}^{H} \mathbf{G}=\alpha \mathbf{I}$, where $\alpha$ changes from 0 to 1.95 (note that $\mathbf{H}^{H} \mathbf{H}-\mathbf{G}^{H} \mathbf{G} \succ \mathbf{0}$ only for $\alpha \leq 1.95$ ). As predicted, the secrecy capacity achieved by the derived $\mathbf{Q}^{*}$ in (50) approaches the standard capacity as $\mathbf{G} \rightarrow \mathbf{0}$. It is interesting to note that even for very small values of $\alpha$, the difference between the standard capacity and secrecy capacity is considerable. 


\section{CONCLUSION}

In this paper, we considered the rank property of the optimal input covariance matrix under an average power constraint for a general MIMO Gaussian wiretap channel, where each node has an arbitrary number of antennas. We obtained necessary and sufficient conditions on the MIMO wiretap channel parameters such that the optimal input covariance matrix is full-rank, and we presented a method for characterizing the resulting covariance matrix as well. When the covariance is not full rank, we showed that there exists an equivalent set of channels and a full-rank transmit covariance that yields the same secrecy capacity as the original scenario.

\section{APPENDIX A}

\section{PROOF OF THEOREM 1}

Recalling from Lemma 3 that $\widehat{b}=\operatorname{rank}\left(\mathbf{Q}^{*}\right)$, we want to show that $\widehat{b} \leq m$. To prove that $\operatorname{rank}\left(\mathbf{Q}^{*}\right)=\widehat{b} \leq m$, we only need to show that there is no $\mathbf{U}_{c} \in \mathbb{C}^{n_{t} \times \widehat{b}}, \mathbf{U}_{c}^{H} \mathbf{U}_{c}=\mathbf{I}$, such that $\mathbf{U}_{c}^{H}\left[\mathbf{H}^{H} \mathbf{H}-\mathbf{G}^{H} \mathbf{G}\right] \mathbf{U}_{c} \succ \mathbf{0}$ and $\widehat{b}>m$. The proof is obtained by contradiction. We assume $\widehat{b}>m$ and show that under this assumption, there exist specific non-zero vectors $\mathbf{e}_{x}$ for which $\mathbf{e}_{x}^{H} \mathbf{U}_{c}^{H}\left[\mathbf{H}^{H} \mathbf{H}-\mathbf{G}^{H} \mathbf{G}\right] \mathbf{U}_{c} \mathbf{e}_{x}=0$, which contradicts the fact that (26) is positive definite.

Denote the eigenvalue decomposition of $\mathbf{H}^{H} \mathbf{H}-\mathbf{G}^{H} \mathbf{G}$ as

$$
\mathbf{H}^{H} \mathbf{H}-\mathbf{G}^{H} \mathbf{G}=\sum_{i=1}^{m} \lambda_{i}^{+} \mathbf{f}_{i} \mathbf{f}_{i}^{H}+\sum_{i=m+1}^{n_{t}} \lambda_{i}^{-} \mathbf{f}_{i} \mathbf{f}_{i}^{H},
$$

where $\lambda_{i}^{+}, \lambda_{i}^{-}$respectively represent positive and non-positive eigenvalues. Then

$$
\mathbf{U}_{c}^{H}\left[\mathbf{H}^{H} \mathbf{H}-\mathbf{G}^{H} \mathbf{G}\right] \mathbf{U}_{c}=\sum_{i=1}^{m} \lambda_{i}^{+} \mathbf{t}_{i} \mathbf{t}_{i}^{H}+\sum_{i=m+1}^{n_{t}} \lambda_{i}^{-} \mathbf{t}_{i} \mathbf{t}_{i}^{H} \succ \mathbf{0},
$$

where $\mathbf{t}_{i}=\mathbf{U}_{c}^{H} \mathbf{f}_{i} \in \widehat{C}^{\widehat{b} \times 1}$. Assuming $\widehat{b}>m$, define $\mathbf{T}^{+}=$ $\left[\mathbf{t}_{1} \ldots \mathbf{t}_{m}\right]$. Let $\mathbf{P}_{\mathbf{T}^{+}}$denote the projection matrix on the column span of $\mathbf{T}^{+}$, and let $\widehat{r}=\operatorname{rank}\left(\mathbf{T}^{+}\right)$. We note that any $\mathbf{t}_{i}$ corresponding to $\lambda_{i}^{-}$can be written as

$$
\mathbf{t}_{i}=\mathbf{P}_{\mathbf{T}^{+}} \mathbf{t}_{i}+\sum_{j=1}^{\widehat{b}-\widehat{r}} v_{i j} \mathbf{e}_{j} \quad i=m+1, \ldots n_{t},
$$

where $\left\{\mathbf{e}_{j}\right\}_{j=1}^{j=\widehat{b}-\widehat{r}}$ is a set of orthonormal basis vectors for $\operatorname{span}\left\{\mathbf{T}^{+}\right\}^{\perp}$, and $v_{i j}$ is a complex scalar. Noting that $\mathbf{e}_{k}^{H} \mathbf{e}_{j}=1$ if $j=k$ and $\mathbf{e}_{k}^{H} \mathbf{e}_{j}=0$ when $j \neq k$, one can easily confirm that

$$
\begin{aligned}
& \mathbf{e}_{k}^{H} \mathbf{U}_{c}^{H}\left[\mathbf{H}^{H} \mathbf{H}-\mathbf{G}^{H} \mathbf{G}\right] \mathbf{U}_{c} \mathbf{e}_{k} \\
&=\sum_{i=m+1}^{i=n_{t}} \lambda_{i}^{-}\left|v_{i k}\right|^{2}>0, \quad k=1, \ldots, \widehat{b}-\widehat{r}
\end{aligned}
$$

Recalling that $\lambda_{i}^{-} \leq 0$, (69) leads to a contradiction since the right side of (69) is zero (after setting $v_{i k}=0$ for non-zero $\lambda_{i}^{-}$), while the left side must be positive. This contradiction comes from the guaranteed existence of non-zero vectors $\left\{\mathbf{e}_{j}\right\}_{j=1}^{j \widehat{b}-\widehat{r}}$ when $\widehat{b}$ is assumed to be bigger than $m$. Thus, $\operatorname{rank}\left(\mathbf{Q}^{*}\right)=\widehat{b} \leq m$.

\section{APPENDIX B}

Proof of LeMma 4

Define $\overline{\mathbf{S}}=\left(\mathbf{H}^{H} \mathbf{H}-\mathbf{G}^{H} \mathbf{G}\right)^{-1}$ and apply the generalized eigenvalue decomposition on the pencil $\left(\overline{\mathbf{S}}^{\frac{1}{2}} \mathbf{H}^{H} \mathbf{H} \overline{\mathbf{S}}^{\frac{1}{2}}+\mathbf{I}, \overline{\mathbf{S}}^{\frac{1}{2}} \mathbf{G}^{H} \mathbf{G} \overline{\mathbf{S}}^{\frac{1}{2}}+\mathbf{I}\right)$ to obtain the invertible generalized eigenvector matrix $\overline{\mathbf{C}}$ and the diagonal generalized eigenvalue matrix $\boldsymbol{\Lambda}_{\overline{\mathrm{s}}}$ as

$$
\begin{aligned}
& \overline{\mathbf{C}}^{H}\left[\overline{\mathbf{S}}^{\frac{1}{2}} \mathbf{H}^{H} \mathbf{H} \overline{\mathbf{S}}^{\frac{1}{2}}+\mathbf{I}\right] \overline{\mathbf{C}}=\boldsymbol{\Lambda}_{\overline{\mathbf{s}}} \\
& \overline{\mathbf{C}}^{H}\left[\overline{\mathbf{S}}^{\frac{1}{2}} \mathbf{G}^{H} \mathbf{G} \overline{\mathbf{S}}^{\frac{1}{2}}+\mathbf{I}\right] \overline{\mathbf{C}}=\mathbf{I} .
\end{aligned}
$$

By subtracting (71) from (70), we have

$$
\overline{\mathbf{C}}^{H} \overline{\mathbf{C}}=\boldsymbol{\Lambda}_{\overline{\mathbf{s}}}-\mathbf{I} \text {. }
$$

Note that from Lemma 1, we have $\boldsymbol{\Lambda}_{\overline{\mathbf{s}}}-\mathbf{I} \succ \mathbf{0}$. Thus, $\overline{\mathbf{C}}$ must be of the form [16]

$$
\overline{\mathbf{C}}=\boldsymbol{\Phi}_{\overline{\mathbf{s}}}\left(\boldsymbol{\Lambda}_{\overline{\mathbf{s}}}-\mathbf{I}\right)^{\frac{1}{2}},
$$

where $\boldsymbol{\Phi}_{\overline{\mathrm{s}}}$ is an unknown unitary matrix. In the following, as we continue the proof, $\boldsymbol{\Phi}_{\overline{\mathrm{s}}}$ is also characterized.

By substituting (73) in (70) and (71), it is revealed that the unitary matrix $\boldsymbol{\Phi}_{\overline{\mathbf{s}}}$ represents the common set of eigenvectors for the matrices $\overline{\mathbf{S}}^{\frac{1}{2}} \mathbf{H}^{H} \mathbf{H} \overline{\mathbf{S}}^{\frac{1}{2}}+\mathbf{I}$ and $\overline{\mathbf{S}}^{\frac{1}{2}} \mathbf{G}^{H} \mathbf{G} \overline{\mathbf{S}}^{\frac{1}{2}}+\mathbf{I}$, and thus both matrices commute. In particular,

$$
\begin{aligned}
\boldsymbol{\Phi}_{\overline{\mathbf{s}}}^{H}\left[\overline{\mathbf{S}}^{\frac{1}{2}} \mathbf{H}^{H} \mathbf{H} \overline{\mathbf{S}}^{\frac{1}{2}}+\mathbf{I}\right] \boldsymbol{\Phi}_{\overline{\mathbf{s}}} & =\boldsymbol{\Lambda}_{\overline{\mathbf{s}}}\left(\boldsymbol{\Lambda}_{\overline{\mathbf{s}}}-\mathbf{I}\right)^{-1} \\
& =\mathbf{I}+\left(\boldsymbol{\Lambda}_{\overline{\mathbf{s}}}-\mathbf{I}\right)^{-1} \\
\boldsymbol{\Phi}_{\overline{\mathbf{s}}}^{H}\left[\overline{\mathbf{S}}^{\frac{1}{2}} \mathbf{G}^{H} \mathbf{G} \overline{\mathbf{S}}^{\frac{1}{2}}+\mathbf{I}\right] \boldsymbol{\Phi}_{\overline{\mathbf{s}}} & =\left(\boldsymbol{\Lambda}_{\overline{\mathbf{s}}}-\mathbf{I}\right)^{-1} .
\end{aligned}
$$

Defining $\mathbf{D}=\left(\boldsymbol{\Lambda}_{\overline{\mathbf{s}}}-\mathbf{I}\right)^{-1}$, from (74), (75) and via straightforward computation, we have

$$
\begin{aligned}
& \mathbf{H}^{H} \mathbf{H}=\overline{\mathbf{S}}^{-\frac{1}{2}} \boldsymbol{\Phi}_{\overline{\mathbf{S}}} \mathbf{D} \boldsymbol{\Phi}_{\overline{\mathbf{s}}}^{H} \overline{\mathbf{S}}^{-\frac{1}{2}} \\
& \mathbf{G}^{H} \mathbf{G}=\overline{\mathbf{S}}^{-\frac{1}{2}} \boldsymbol{\Phi}_{\overline{\mathbf{s}}}(\mathbf{D}-\mathbf{I}) \boldsymbol{\Phi}_{\overline{\mathbf{s}}}^{H} \overline{\mathbf{S}}^{-\frac{1}{2}},
\end{aligned}
$$

which proves (39) and (40).

\section{APPENDIX C \\ PROOF OF THEOREM 3}

Recall from Lemma 3 and (24) that $\widehat{b}=\operatorname{rank}\left(\mathbf{Q}^{*}\right)$ and that the generalized eigenvalue decomposition of the pencil $\left(\mathbf{Q}^{* \frac{1}{2}} \mathbf{H}^{H} \mathbf{H} \mathbf{Q}^{* \frac{1}{2}}+\mathbf{I}, \mathbf{Q}^{* \frac{1}{2}} \mathbf{G}^{H} \mathbf{G Q}^{* \frac{1}{2}}+\mathbf{I}\right)$ can be rewritten as

$$
\begin{aligned}
& \widehat{\mathbf{C}}^{H}\left[\mathbf{Q}^{* \frac{1}{2}} \mathbf{H}^{H} \mathbf{H} \mathbf{Q}^{* \frac{1}{2}}+\mathbf{I}\right] \widehat{\mathbf{C}}=\left[\begin{array}{cc}
\widehat{\boldsymbol{\Lambda}}_{1} & \mathbf{0} \\
\mathbf{0} & \mathbf{I}
\end{array}\right] \\
& \widehat{\mathbf{C}}^{H}\left[\mathbf{Q}^{* \frac{1}{2}} \mathbf{G}^{H} \mathbf{G Q}^{* \frac{1}{2}}+\mathbf{I}\right] \widehat{\mathbf{C}}=\left[\begin{array}{ll}
\mathbf{I} & \mathbf{0} \\
\mathbf{0} & \mathbf{I}
\end{array}\right]
\end{aligned}
$$


where $\widehat{\mathbf{C}}=\left[\widehat{\mathbf{C}}_{1} \widehat{\mathbf{C}}_{2}\right]$ is the generalized eigenvector matrix, $\widehat{\mathbf{C}}_{1} \in \mathbb{C}^{n_{t} \times \widehat{b}}$ corresponds to $\widehat{\boldsymbol{\Lambda}}_{1}$, and $\widehat{\boldsymbol{\Lambda}}_{1}$ is a $\widehat{b} \times \widehat{b}$ diagonal matrix with diagonal elements representing generalized eigenvalues greater that 1 . We note that only the generalized eigenvalues bigger than 1 contribute to the secrecy capacity, as shown in (10).

Rewrite (78), this time only for the generalized eigenvectors corresponding to eigenvalues bigger than 1 :

$$
\begin{aligned}
& \widehat{\mathbf{C}}_{1}^{H}\left[\mathbf{Q}^{* \frac{1}{2}} \mathbf{H}^{H} \mathbf{H} \mathbf{Q}^{* \frac{1}{2}}+\mathbf{I}\right] \widehat{\mathbf{C}}_{1}=\widehat{\mathbf{\Lambda}}_{1} \\
& \widehat{\mathbf{C}}_{1}^{H}\left[\mathbf{Q}^{* \frac{1}{2}} \mathbf{G}^{H} \mathbf{G Q}^{* \frac{1}{2}}+\mathbf{I}\right] \widehat{\mathbf{C}}_{1}=\mathbf{I} .
\end{aligned}
$$

Similar to what we did in (21)-(26), denote the singular value decomposition of $\widehat{\mathbf{C}}_{1}$ as

$$
\widehat{\mathbf{C}}_{1}=\mathbf{U}_{c} \mathbf{D}_{c} \mathbf{V}_{c}^{H},
$$

where $\mathbf{U}_{c}$ is a $n_{t} \times \widehat{b}$ matrix whose columns are an orthonormal basis for $\operatorname{span}\left\{\widehat{\mathbf{C}}_{1}\right\}, \mathbf{U}_{c}^{H} \mathbf{U}_{c}=\mathbf{I}, \mathbf{D}_{c}$ is a $\widehat{b} \times \widehat{b}$ positive definite diagonal matrix, and $\mathbf{V}_{c}$ is a unitary matrix. Recall from lemma 2 that $\operatorname{span}\left\{\widehat{\mathbf{C}}_{1}\right\}=\operatorname{span}\left\{\mathbf{Q}^{*}\right\}$, then $\mathbf{Q}^{*}$ can be written as

$$
\mathbf{Q}^{*}=\mathbf{U}_{c} \mathbf{Q}_{e q}^{*} \mathbf{U}_{c}^{H},
$$

where $\mathbf{Q}_{e q}^{*} \in \mathbb{C}^{\widehat{b} \times \widehat{b}}$ and $\mathbf{Q}_{e q}^{*} \succ \mathbf{0}$. Using (80) and (81) in (79), we have

$$
\begin{aligned}
& \mathbf{V}_{c} \mathbf{D}_{c}\left[\mathbf{Q}_{e q}^{* \frac{1}{2}} \mathbf{U}_{c}^{H} \mathbf{H}^{H} \mathbf{H} \mathbf{U}_{c} \mathbf{Q}_{e q}^{* \frac{1}{2}}+\mathbf{I}\right] \mathbf{D}_{c} \mathbf{V}_{c}^{H}=\widehat{\mathbf{\Lambda}}_{1} \\
& \mathbf{V}_{c} \mathbf{D}_{c}\left[\mathbf{Q}_{e q}^{* \frac{1}{2}} \mathbf{U}_{c}^{H} \mathbf{G}^{H} \mathbf{G} \mathbf{U}_{c} \mathbf{Q}_{e q}^{* \frac{1}{2}}+\mathbf{I}\right] \mathbf{D}_{c} \mathbf{V}_{c}^{H}=\mathbf{I} .
\end{aligned}
$$

The equations in (82) correspond to the generalized eigenvalue decomposition of the pencil $\left(\mathbf{Q}_{e q}^{* \frac{1}{2}} \mathbf{H}_{e q}^{H} \mathbf{H}_{e q} \mathbf{Q}_{e q}^{* \frac{1}{2}}+\mathbf{I}, \mathbf{Q}_{e q}^{* \frac{1}{2}} \mathbf{G}_{e q}^{H} \mathbf{G}_{e q} \mathbf{Q}_{e q}^{* \frac{1}{2}}+\mathbf{I}\right), \quad$ where $\mathbf{H}_{e q}=\mathbf{H U}_{c}$ and $\mathbf{G}_{e q}=\mathbf{G U}_{c}$, the generalized eigenvector matrix is $\mathbf{D}_{c} \mathbf{V}_{c}^{H}$ and the diagonal generalized eigenvalue matrix is $\widehat{\Lambda}_{1}$.

Note that for the wiretap channel $\left(\mathbf{H}_{e q}, \mathbf{G}_{e q}\right)$, the number of transmit antennas is less than that of $(\mathbf{H}, \mathbf{G})$, i.e. $\widehat{b}<n_{t}$; this indicates that $\mathcal{C}_{s e c}\left(\mathbf{H}_{e q}, \mathbf{G}_{e q}, P_{t}\right) \leq \mathcal{C}_{s e c}\left(\mathbf{H}, \mathbf{G}, P_{t}\right)$. However, (82) shows the achievablity of the same set of generalized eigenvalues bigger than $1, \widehat{\boldsymbol{\Lambda}}_{1}$, for the wiretap channel $\left(\mathbf{H}_{e q}, \mathbf{G}_{e q}\right)$ when the input covariance matrix is $\mathbf{Q}_{e q}^{*} \succ \mathbf{0}$. Consequently, $\mathbf{Q}_{e q}^{*}$ is the optimal full-rank input covariance matrix for the equivalent wiretap channel that achieves $\mathcal{C}_{s e c}\left(\mathbf{H}_{e q}, \mathbf{G}_{e q}, P_{t}\right)=$ $\mathcal{C}_{\text {sec }}\left(\mathbf{H}, \mathbf{G}, P_{t}\right)$. that

By subtracting the two equalities in (82), one can easily show $\mathbf{V}_{c} \mathbf{D}_{c} \mathbf{Q}_{e q}^{* \frac{1}{2}}\left[\mathbf{H}_{e q}^{H} \mathbf{H}_{e q}-\mathbf{G}_{e q}^{H} \mathbf{G}_{e q}\right] \mathbf{Q}_{e q}^{* \frac{1}{2}} \mathbf{D}_{c} \mathbf{V}_{c}^{H}=\widehat{\mathbf{\Lambda}}_{1}-\mathbf{I} \succ \mathbf{0}$, which results that in the equivalent wiretap channel we have $\mathbf{H}_{e q}^{H} \mathbf{H}_{e q}-\mathbf{G}_{e q}^{H} \mathbf{G}_{e q} \succ \mathbf{0}$.

\section{REFERENCES}

[1] A. Wyner, "The wire-tap channel," Bell. Syst. Tech. J., vol. 54, no. 8 pp. 1355-1387, Jan. 1975.

[2] S. K. Leung-Yan-Cheong and M. E. Hellman, "The Gaussian wire-tap channel," IEEE Trans. Inf. Theory, vol. 24, pp. 451-456, Jul. 1978.

[3] F. Oggier and B. Hassibi, "The secrecy capacity of the MIMO wiretap channel," in Proc. IEEE Int. Symp. Inf. Theory, Toronto, ON, Canada, Jul. 2008, pp. 524-528.

[4] A. Khisti and G. Wornell, "Secure transmission with multiple antennas II: The MIMOME wiretap channel," IEEE Trans. Inf. Theory, vol. 56, no. 11, pp. 5515-5532, 2010.

[5] T. Liu and S. Shamai (Shitz), "A note on secrecy capacity of the multiantenna wiretap channel," IEEE Trans. Inf. Theory, vol. 55, no. 6, pp. $2547-2553,2009$

[6] H. Weingarten, Y. Steinberg, and S. Shamai (Shitz), "The capacity region of the Gaussian multiple-input multipleoutput broadcast channel," IEEE Trans. Inf. Theory, vol. 52, no. 9, pp. 3936-3964, 2006.

[7] R. Bustin, R. Liu, H. V. Poor, and S. Shamai (Shitz), "A MMSE approach to the secrecy capacity of the MIMO Gaussian wiretap channel," EURASIP J. Wireless Commun. Netw., vol. 2009, 2009 Article ID 370970, 8 pp.

[8] L. Zhang, R. Zhang, Y. Liang, Y. Xin, and S. Cui, "On the relationship between the multi-antenna secrecy communications and cognitive radio communications," IEEE Trans. Commun., vol. 58, no. 6, pp. 1877-1886, Jun. 2010.

[9] S. A. A. Fakoorian and A. L. Swindlehurst, "Optimal power allocation for the GSVD-based beamforming in the MIMO Gaussian wiretap channel," in Proc. IEEE Int. Symp. Inform. Theory (ISIT), Jul. 2012, pp. 2321-2325.

[10] J. Li and A. P. Petropulu, "Transmitter optimization for achieving secrecy capacity in Gaussian MIMO wiretap channels," IEEE Trans. Inf. Theory [Online]. Available: http://arxiv.org/PScache/arxiv/pdf/ 0909/0909.2622v1.pdf, submitted for publication

[11] A. Khisti and G. Wornell, "Secure transmission with multiple antennas I: The MISOME wiretap channel," IEEE Trans. Inf. Theory, vol. 56, no. 7, pp. 3088-3104, 2010.

[12] S. Shafiee and S. Ulukus, "Towards the secrecy capacity of the Gaussian MIMO wire-tap channel: The 2-2-1 channel," IEEE Trans. on Inf. Theory, vol. 55, no. 9, pp. 4033-4039, Sep. 2009.

[13] S. A. A. Fakoorian and A. L. Swindlehurst, "Rank property of the MIMO Gaussian wiretap channel with an average power constraint," in Proc. 46th Asilomar Conf. Signals, Syst. Comput., Nov. 2012

[14] S. Loyka and C. D. Charalamboust, "On optimal signaling over secure MIMO channels," in Proc. IEEE Int. Symp. Inf. Theory (ISIT), Jul. 2012, pp. 443-447.

[15] R. Liu, T. Liu, H. V. Poor, and S. Shamai, "Multiple-input multipleoutput Gaussian broadcast channels with confidential messages," IEEE Trans. Inf. Theory, vol. 56, no. 9, pp. 4215-4227, 2010.

[16] R. A. Horn and C. R. Johnsonc, Matrix Analysis. Cambridge, U.K.: Cambridge Univ. Press, 1999.

[17] C. F. V. Loan, "Generalizing the singular value decomposition," SIAM J. Numer. Anal., vol. 13, no. 1, pp. 76-83, Mar. 1973.

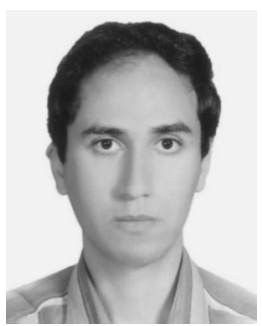

S. Ali. A. Fakoorian (S'08) received the B.S. and M.S. degrees, both in electrical engineering, from Amirkabir University of Technology, Tehran, Iran, in 2002 and 2005, respectively.

$\mathrm{He}$ is currently working towards the Ph.D. degree at the Department of Electrical Engineering and Computer Science, University of California, Irvine, CA, USA. His research interests lie in multiple-user wireless networks, signal processing, and information theory.

Mr. Fakoorian is a student member of CPCC and the IEEE Communications and Signal Processing Societies. 


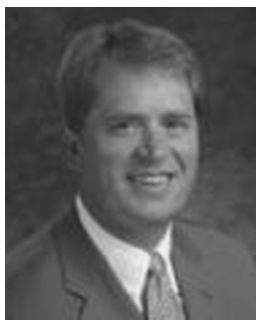

A. Lee Swindlehurst (F'04) received the B.S (summa cum laude) and M.S. degrees in electrical engineering from Brigham Young University, Provo, UT, USA, in 1985 and 1986, respectively, and the $\mathrm{Ph} . \mathrm{D}$. degree in electrical engineering from Stanford University, Stanford, CA, USA, in 1991.

From 1986 to 1990, he was with ESL, Inc., Sunnyvale, CA, USA, where he was involved in the design of algorithms and architectures for several radar and sonar signal processing systems. He was on the faculty of the Department of Electrical and Computer Engineering at Brigham Young University from 1990 to 2007, where he was a Full Professor and served as Department Chair from 2003 to 2006. During 1996-1997, he held a joint appointment as a visiting scholar at both Uppsala University, Uppsala, Sweden, and at the Royal Institute of Technology, Stockholm, Sweden. From 2006 to 2007, he was on leave working as Vice-President of Research for ArrayComm LLC, San Jose, CA, USA. He is currently a Professor of electrical engineering and computer science with the University of California at Irvine, CA, USA. His research interests include sensor array signal processing for radar and wireless communications, detection and estimation theory, and system identification, and he has over 200 publications in these areas.

Dr. Swindlehurst is a past Secretary of the IEEE Signal Processing Society, past Editor-in-Chief of the IEEE Journal OF SELECTED TOPICS IN SigNAL PROCESSING, and past member of the Editorial Boards for the EURASIP Journal on Wireless Communications and Networking, the IEEE Signal Processing Magazine, and the IEEE TRANSACTIONS ON SignAL PROCESSING. He is a recipient of several paper awards: the 2000 IEEE W. R. G. Baker Prize Paper Award, the 2006 and 2010 IEEE Signal Processing Society Best Paper Award, and the 2006 IEEE Communications Society Stephen O. Rice Prize in the Field of Communication Theory, and he is coauthor of a paper that received the IEEE Signal Processing Society Young Author Best Paper Award in 2001. 